\title{
Electrons in superheavy quasimolecules
}

\author{
Gerhard Soff and Walter Greiner \\ Department of Physics and Astronomy, Nuclear Physics Division, Vanderbilt University, Nashville, Tennessee 37203 \\ and Physics Division, Oak Ridge National Laboratory, Oak Ridge, Tennessee 37830 \\ Wilfried Betz and Berndt Müller \\ Institut für Theoretische Physik, Universität Frankfurt am Main, West Germany
}

(Received 7 August 1978)

\begin{abstract}
Binding energies and wave functions of inner-shell electronic states in superheavy quasimolecules with $\left(Z_{p}+Z_{t}\right) \alpha>1$ are calculated. Ionization during a collision of very heavy ions is investigated within a molecular basis generated by the solutions of the two-center Dirac equation. Transitions to vacant bound states as well as direct excitation to the continuum are taken into account. We present theoretical values for the ionization probability as a function of impact parameter, bombarding energy, and combined nuclear charge. Our computed results are compared with recent experimental data. It is suggested that relativistic binding energies of electrons in superheavy quasimolecules can be determined experimentally via the impactparameter dependence of ionization and the anisotropy of quasimolecular radiation.
\end{abstract}

\section{INTRODUCTION}

The motivation for our investigations is to lay the grounds for a possible spectroscopy of strongly bound electronic states in superheavy quasimolecules. In such systems inner-shell electrons move quasi adiabatically in the Coulomb field of two scattering charge centers, where the twocenter distance $R(t)$ changes as function of collision time $t$. Also, in the collision of very heavy ions, such transient systems can be formed in which even the radius of the quasimolecular $K$ shell is much larger than the separation $R$ of the scattering nuclei. In these superheavy quasiatoms the energy and wave function of electrons are determined by the united Coulomb field of both nuclei. In collisions of $\mathrm{U}$ on $\mathrm{U}$ a quasiatom is created for $R \ll 500 \mathrm{fm}$. At a lab velocity of $v_{\text {ion }}$ $=0.1 \mathrm{c}$, the corresponding electrons move for a time of $\tau=10^{-20} \mathrm{sec}$ in an external field with $Z$ $=Z_{p}+Z_{t}=184$, where $Z_{p}$ and $Z_{t}$ denote the projectile charge and target charge, respectively. Such superheavy atoms could be investigated experimentally if it would be possible to synthesize stable nuclei in the region $Z=114$ or $Z=164$, as predicted theoretically. However, all corresponding experimental attempts remained unsuccessful up to now. ${ }^{1}$ Also, in nature no superheavy elements could be detected. Therefore the only possibility to study the behavior of electrons in strong electric fields of about $E=10^{19} \mathrm{~V} / \mathrm{cm}$ is the formation of quasimolecules and quasiatoms in collisions of very heavy ions.

In heavy-ion collisions we can extend the traditional domain of atomic physics (concerning the charge number) by almost a factor of 2 from the region of known elements $(Z \leqslant 107)$ up to $Z=190$ in a collision of a $U$ projectile on a Cf target.

In the following we consider the binding energies of electrons in superheavy atoms. For Fermium $(Z=100)$ the binding energy of the strongest bound electron amounts to $E_{1 s}=-141 \mathrm{keV}$ with an agreement between theory and experiment of about $10 \mathrm{eV}^{2,3}$ In the corresponding calculations one has to take into account the finite size of the nucleus, the electron-electron interaction treated within the Hartree- ${ }^{\top}$ uck formalism, and quantum electrodynamical orrections like vacuum polarization, self-energy, and magnetic effects. When those calculations are extended to the superheavy region, ${ }^{4-9}$ the $1 s$ binding energy increases drastically as function of nuclear charge. At $Z=150$ it reaches the value of the electronic rest mass; for $Z=173$ the binding is twice as large. For still higher central charges the $1 s$ state dives into the negative energy continuum of the Dirac equa tion and becomes a resonance imbedded in this continuum. ${ }^{10-12}$ The corresponding critical charge for the $2 p_{1 / 2}$ state is about $Z=185$. These extremely strong binding energies, which are comparable to the electronic rest mass $m_{e} c^{2}$, necessitate a relativistic treatment of the investigated problem. Instead of the Schrödinger equation we have to use its relativistic counterpart, the Dirac equation, for the evaluation of electronic states. Besides the stronger binding, the relativistic ef fects manifest themselves in a dramatic "fine structure" splitting $\Delta E=E\left(2 p_{3 / 2}\right)-E\left(2 p_{1 / 2}\right)$, which again is of the order of $m_{e} c^{2}$. We conclude that concerning the binding energies superheavy atoms would help to extend the energy range of traditional atomic physics by an order of magni- 
tude.

Furthermore, the quantum electrodynamical corrections in superheavy quasimolecules can no longer be evaluated in the usual perturbation expansion in $Z \alpha$, because the electromagnetic coupling constant becomes larger than one, $\left(Z_{p}\right.$ $\left.+Z_{t}\right) \alpha>1$. Calculations of vacuum polarization $^{13,14}$ and self-energy ${ }^{15}$ in all orders of $Z \alpha$, however, have shown that these effects amount only to about $1 \%$ of the total binding energy even for $Z \sim 170$, and therefore can be neglected at present. In order to account for the electronelectron interaction, numerous calculations have been performed within the framework of the Hartree-Fock formalism. ${ }^{7,8,16-23}$ By this the identification of eventual stable superheavy elements should be facilitated. In the following $d y-$ namical calculations, however, we will use a basis in which the electron-electron interaction ${ }^{89}$ is not prediagonalized. Transitions between inner electronic states induced by this interaction are negligible, because the relaxation time of electrons against the adjustment of the orbitals due to electron-electron interaction is large compared with the collision time of the investigated scattering systems. ${ }^{24}$ Thus self-consistent calculations are clearly not called for; they may even not be an improvement. Also the diagonal matrix elements of the electron-electron interaction remain unconsidered in our calculations, because we have to deal with a system of undetermined degree of ionization. The diagonal part of the electronelectron interaction results in a shift of the boundstate energies without modification of the wave functions. The shifts amount to about $10 \%$ for the $1 s \sigma$ state and will slightly enhance the ionization probabilities. Its precise magnitude can only be determined when more complete information on the electronic configuration during the collision is available.

In the following we will propose various pos sibilities ${ }^{25,26}$ to experimentally determine binding energies of inner electronic states in systems up to $Z=190$ : (a) the impact-parameter dependence of ionization mediated by the radial and rotational coupling of the scattering nuclei with the electrons [here the asymptotic $(t \rightarrow+\infty)$ radiation (e.g., $K_{\alpha}$ radiation) in the separated single atoms after the collision must be measured in coincidence with the ion scattering angle], (b) the $Z$ dependence of ionization in central collisions, (c) the distribution of the ionized electrons ${ }^{27,28}$ in the continuum final states ( $\delta$ electrons) with respect to energy and to the dependence on impact parameter and (d) the frequency and angular distribution of emitted quasimolecular radiation during the collision. ${ }^{29-35,53,54,77,78}$
After this introduction we will briefly describe the formalism of ionization. Then we discuss in detail the calculation of bound states within the framework of the two-center Dirac equation, the corresponding coupling matrix elements, and the validity of the monopole approximation, in which one considers only the spherically symmetric part of the two-center potential. We continue to present the evaluation of continuum states and the couplings to bound states. As a first example of a dynamical process we will give the transition probability of bound electrons to the positive continuum as a function of distance $R$ between the two centers during the collision, the impact parameter $b$, the total charge $Z_{p}+Z_{t}$, and the kinetic ion energy $E_{10 n}$. The obtained theoretical results will be compared with recent experimental data da-39, $^{32,85}$ concerning ionization in superheavy quasimolecules. As a function of the same parameters we will investigate the coupling between bound states within the coupled channel formalism.

As a next step towards a full-scale spectroscopy we present the theoretical $\delta$-electron distribution.

As an example of the influence of rotational coupling we consider the $2 p_{1 / 2} \sigma$ ionization ${ }^{40,41}$ in the $\mathrm{Pb}-\mathrm{Pb}$ system. Before we finally draw our conclusions we will briefly discuss the background contribution to ionization and quasimolecular radiation caused by nuclear Coulomb excitation. ${ }^{42}$

\section{BASIC FORMALISM FOR INNER-SHELL IONIZATION IN COLLISION OF VERY HEAVY IONS}

The dynamical processes, such as ionization, occurring during quasimolecular collisions are described by the time-dependent Dirac equation $(\hbar=c=1)$ :

$$
i \frac{\partial \psi(\overrightarrow{\mathrm{r}}, t)}{\partial t}=H_{\text {Dirac }} \psi(\overrightarrow{\mathrm{r}}, t) .
$$

For practical purposes, the wave function $\psi$ is expanded in a complete set of basis states ${ }^{73}$ :

$$
\psi(\overrightarrow{\mathrm{r}}, t)=\sum_{n} a_{n}(t) \phi_{n}[\overrightarrow{\mathrm{r}}, R(t)] \exp \left(-i \int_{-\infty}^{t} E_{n} d t^{\prime}\right) \text {. }
$$

Since binding energies and wave functions change strongly as a function of the two-center distance $R$, the proper choice in collisions at nonrelativistic energies is the molecular basis, given by the solutions $\phi_{n}$ of the stationary two-center Dirac equation $^{43}, 44$ :

$$
\left(\vec{\alpha} \overrightarrow{\mathrm{p}}+\beta m_{e}-\frac{Z_{1} e^{2}}{\left|\overrightarrow{\mathrm{r}}-\frac{1}{2} \overrightarrow{\mathrm{R}}\right|}-\frac{Z_{2} e^{2}}{\left|\overrightarrow{\mathrm{r}}+\frac{1}{2} \overrightarrow{\mathrm{R}}\right|}-E_{n}(R)\right) \phi_{n}(\overrightarrow{\mathrm{r}}, R)
$$$$
=0 \text {. }
$$ 
The two-center wave functions $\phi_{n}$ depend on time parametrically. We assume the nuclear motion to follow a Coulomb trajectory, given by a Rutherford hyperbola, thereby neglecting possible effects of nuclear forces, ${ }^{45}$ electron shielding, ${ }^{46,47}$ and vacuum polarization. ${ }^{48,49}$ We briefly summarize the Rutherford hyperbola parametrization:

$$
\begin{aligned}
& R=a(\epsilon \cosh \xi+1), \\
& t=\left(M_{\text {red }} a^{3} / Z_{1} Z_{2} e^{2}\right)^{1 / 2}(\epsilon \sinh \xi+\xi), \\
& t h=\left(1+b^{2} / a^{2}\right)^{1 / 2}, \quad a=Z_{1} Z_{2} e^{2} / 2 E_{\text {c.m. }} .
\end{aligned}
$$$$
\text { with }
$$

$E_{\text {c.m. }}$ is the center-of-mass energy of the ions and $M_{\text {red }}$ the reduced mass of the system:

$$
M_{\mathrm{red}}=M_{1} M_{2} /\left(M_{1}+M_{2}\right) \text {. }
$$

The distance of closest approach $R_{\min }$ between both ions during the collision and the instantaneous radial ion velocity $\dot{R}$ on a given trajectory with impact parameter $b$ are determined by

$$
\begin{aligned}
& R_{\min }=a+\left(a^{2}+b^{2}\right)^{1 / 2}, \\
& \dot{R}=\left(v_{\infty} / R\right)\left[\left(R-R_{\min }\right)\left(R+R_{\min }-2 a\right)\right]^{1 / 2},
\end{aligned}
$$

with $v_{\infty}=\left(2 E_{\mathrm{c} . \mathrm{m} .} / M_{\mathrm{red}}\right)^{1 / 2}$.

$b$ is related to the center-of-mass scattering angle $\theta_{\text {c.m. by }}$

$$
b=a \cot \left(e_{\text {c.m. }} / 2\right) \text {. }
$$

Upon substitution of the ansatz (2) into Eq. (1), one is left with an infinite system of coupled channel equations $^{25,50,73}$ :

$$
\begin{aligned}
\dot{a}_{f}(t)=-\sum_{i \neq f} a_{i}(t)\left\langle\phi_{f}\left|\frac{\partial}{\partial t}\right| \phi_{i}\right\rangle \\
\times \exp \left(i \int_{-\infty}^{t} d t^{\prime}\left(E_{f}-E_{i}\right)\right) .
\end{aligned}
$$

Note that the summation over $i$ includes the continuum states. In our subsequent calculations we split the $\partial / \partial t$ operator into two parts, the radial and the rotational coupling:

$$
\frac{\partial}{\partial t}=\dot{R} \frac{\partial}{\partial R}-i \vec{\omega} \vec{j} \text {. }
$$

Here $\vec{\omega}$ is the angular velocity of the internuclear axis and $\vec{j}$ is the electron angular momentum operator. The two operators exhibit different angular momentum selection rules: the radial coupling acts between states with the same angular momentum projection $\mu$ along the axis, connecting both nuclei ( $z$ axis), whereas the rotational term couples states with $\mu$ differing by \pm 1 . The full set of coupled first-order differential equations (9) is equivalent to the time-dependent Dirac equation.
However, in practical calculations of transitions between bound states one has to restrict to a finite subset of $\left\{\phi_{n}\right\}$. Furthermore, excitations to the continuum states have been treated only in firstorder time-dependent perturbation theory, i.e., taking $a_{i}(t)=1$.

In this approximation the complex amplitude becomes

$$
\begin{aligned}
a_{f}(t)=- & \int_{-\infty}^{t} d t^{\prime}\left\langle\phi_{f}\left|\frac{\partial}{\partial t^{\prime}}\right| \phi_{i}\right\rangle \\
& \times \exp \left(i \int_{-\infty}^{t^{\prime}} d t^{\prime \prime}\left[E_{f}-E_{i}\left(t^{\prime \prime}\right)\right]\right)
\end{aligned}
$$

In the single-electron approximation, the energy $E_{f}$ of the continuum electron states does not depend on time.

The total cross section for direct ionization is obtained by an integration over all impact parameters and all final states:

$$
\begin{aligned}
\sigma & =2 \pi \int_{0}^{\infty} P(b) b d b \\
& =2 \pi\left(2 j_{i}+1\right) \int_{0}^{\infty} b d b \int_{m_{e} e^{c^{2}}}^{\infty} d E_{f}\left|a_{f}(t=\infty)\right|^{2} .
\end{aligned}
$$

The statistical factor $2 j_{i}+1$ accounts for the occupation number of the initial state. Note that the number of created vacancies per collision $P(b)$ is normalized to $P_{\max }=2 j_{i}+1$ and therefore is not a probability in the strict sense. Before presenting results on inner-shell ionization we shall, in the next sections, discuss the calculation of the relativistic two center bound-state and continuum wave functions and the corresponding coupling matrix elements.

\section{BOUND STATE SOLUTIONS OF THE TWO-CENTER DIRAC EQUATION (TCDE)}

In this section we find solutions of the stationary Dirac equation for a two-center potential. We start with the representation of the Dirac equation in spherical coordinates:

$$
\begin{aligned}
{\left[i \gamma _ { 5 } \sigma _ { r } \left(\frac{\partial}{\partial r}\right.\right.} & \left.+\frac{1}{r}-\frac{\beta}{r} \hat{K}\right)-\frac{Z_{1} e^{2}}{\left|\overrightarrow{\mathrm{r}}-\frac{1}{2} \overrightarrow{\mathrm{R}}\right|} \\
& \left.-\frac{Z_{2} e^{2}}{\left|\overrightarrow{\mathrm{r}}+\frac{1}{2} \overrightarrow{\mathrm{R}}\right|}+\beta m_{e}\right] \phi_{\mu}(\overrightarrow{\mathrm{r}})=E \phi_{\mu}(\overrightarrow{\mathrm{r}}) .
\end{aligned}
$$

The spin-orbit operator is defined through the relation

$$
\hat{K} \equiv \beta(\vec{\sigma} \vec{I}+1) .
$$

It is known that the two-center Dirac equation is more difficult to handle than its nonrelativistic counterpart, since it is not separable in any orthogonal coordinate system. The most accurate and flexible approach is based on a multipole ex- 
pansion of the wave function 55,56

$$
\phi_{\mu}(\vec{r})=\sum_{\kappa} \phi_{\mu k}(\vec{r})=\sum_{\kappa}\left(\begin{array}{ll}
g_{\kappa}(r) & X_{\kappa}^{\mu} \\
i f_{\kappa}(r) & X_{-\kappa}^{\mu}
\end{array}\right) .
$$

$f_{k}(r)$ and $g_{k}(r)$ are the radial wave functions, and the spinor spherical harmonics are given by

$$
X_{\kappa}^{\mu}=\sum_{m= \pm 1 / 2}\left(l, \frac{1}{2}, j, \mu-m, m\right) Y_{l}^{\mu-m}(\vartheta, \varphi) \cdot X^{m} .
$$

$-\kappa$ is the eigenvalue of $\hat{K}$ and connected to the angular momentum through

$$
\kappa=\begin{array}{cl}
l & \text { for } j=l-\frac{1}{2} \\
-l-1 & \text { for } j=l+\frac{1}{2}
\end{array},
$$

$j=|\kappa|-\frac{1}{2}$ being the total angular momentum. The magnetic quantum number $\mu$ is the projection of the total angular momentum on the axis connecting the two nuclei ( $z$ axis). After expanding the two center potential into multipoles

$$
V(\overrightarrow{\mathrm{r}}, R)=\sum_{l=0}^{\infty} V_{l}(r, R) P_{l}(\cos \vartheta),
$$

where for $r<R / 2$,

$$
V_{l}(r, R)=\frac{-2 Z_{1} e^{2}}{R}\left(\frac{2 r}{R}\right)^{l}-\frac{2 Z_{2} e^{2}}{R}\left(\frac{-2 r}{R}\right)^{l}
$$

and where for $r \geqslant R / 2$,

$$
V_{l}(r, R)=-\frac{Z_{1} e^{2}}{r}\left(\frac{R}{2 r}\right)^{l}-\frac{Z_{2} e^{2}}{r}\left(\frac{-R}{2 r}\right)^{l},
$$

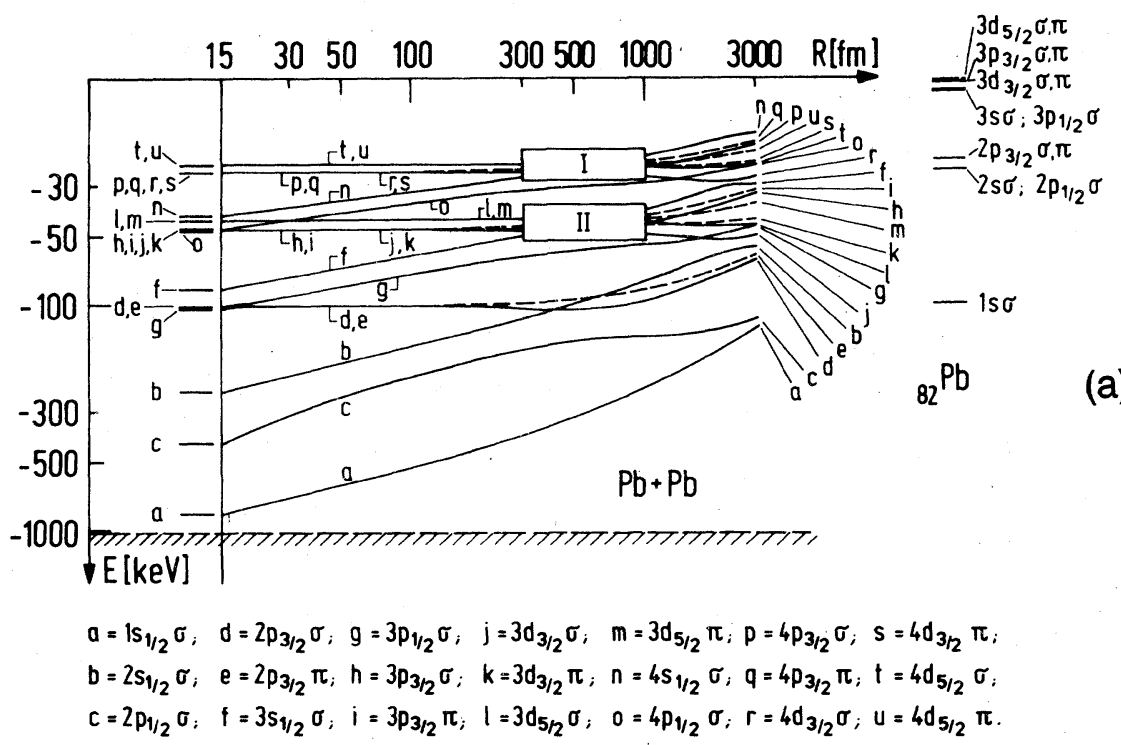

FIG. 1. Relativistic correlation diagram for the $\mathrm{Pb}-\mathrm{Pb}$ system. Binding energies for the lowest $\sigma$ (solid lines) and $\pi$ (dashed lines) states as function of two-center distance $R$. The binding energies of the strongest bound states are given on a linear scale in Fig. 1(b). The energy levels in rectangles I and II are shown separately in Figs. 1 (c) and 1 (d).

(b) 
(c)

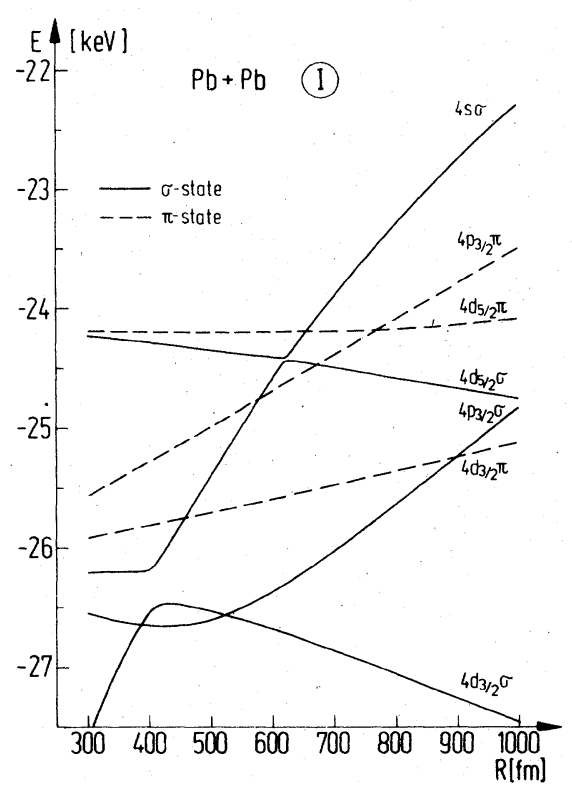

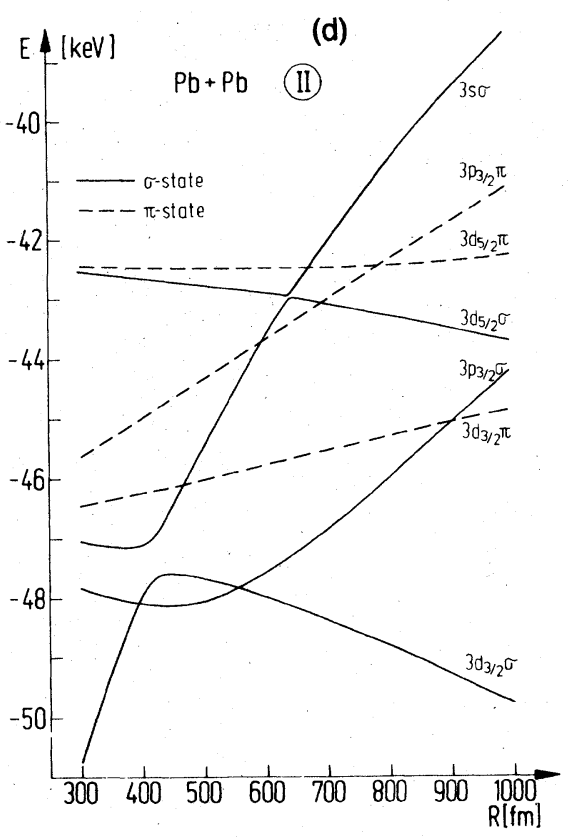

FIG. 1. (Continued)

the coupled radial equations read

$$
\begin{aligned}
\frac{d}{d r} g_{\kappa}(r)= & (E+m) f_{\kappa}(r)-\frac{\kappa+1}{r} g_{\kappa}(r) \\
& -\sum_{\bar{\kappa}, l} f_{\bar{k}}(r) V_{l}(r, R) A_{(-\kappa, l,-\bar{k}}^{\mu}, \\
\frac{d}{d r} f_{\kappa}(r)= & \frac{\kappa-1}{r} f_{\kappa}(r)-(E-m) g_{\kappa}(r) \\
& +\sum_{\bar{\kappa}, l} g_{\bar{\kappa}}(r) V_{l}(r, R) A_{(\kappa, l, \bar{\kappa})}^{\mu} .
\end{aligned}
$$

The coefficients $A_{(k, l, \bar{k})}^{\mu}=\left\langle X_{k}^{\mu}\left|P_{l}\right| X \frac{\mu}{k}\right\rangle$ are easily determined by angular momentum algebra.

It is clear that this representation is not limited to the case of point-like nuclei; any axially symmetric potential $V(\overrightarrow{\mathrm{r}}, R)$ can be treated after a - multipole expansion,

$V_{l}(r, R)=-\frac{2 l+1}{2} \int_{0}^{\pi} d \cos \vartheta P_{l}(\cos \vartheta) V(\overrightarrow{\mathrm{r}}, R)$,

if the requirement $\lim _{l \rightarrow \infty} V_{l}(r, R)=0$ is satisfied for (almost) every $r$ r.

The sum in Eq. (19) has been truncated at a sufficiently large angular momentum $j_{\max }$. The remaining $2\left(2 j_{\max }+1\right)$ coupled differential equations can be solved by a five-point Adams integration code. ${ }^{57}$ The energy eigenvalues are determined by iteration, which is stopped when the change of the energy value is less than $10^{-14}$. In this case the accuracy of the two-center wave function is better than $3 \times 10^{-3}$. The adiabatic correlation diagram for the U-U system has been published elsewhere. ${ }^{43}, 44$

As an example of a relativistic correlation diagram we show in Fig. 1 the binding energies of several bound states in the $\mathrm{Pb}-\mathrm{Pb}$ system. In order to point out the different dependence of energy eigenvalues on two-center distance $R$, we have chosen various representations of the adia batic $\mathrm{Pb}+\mathrm{Pb}$ correlation diagram. In Fig. 1(a) we plot on a double logarithmic scale the 21 lowest $\sigma$ (solid lines) and $\pi$ (dashed lines) states between $R=15 \mathrm{fm}$ and $R=3000 \mathrm{fm}$. The relativistic splittings between the states $2 p_{3 / 2} \sigma-2 p_{1 / 2} \sigma$ of 316.6 $\mathrm{keV}, 3 p_{3 / 2} \sigma-3 p_{1 / 2} \sigma$ of $59.5 \mathrm{keV}$, and $4 p_{3 / 2} \sigma$ $-4 p_{1 / 2} \sigma$ of $21 \mathrm{keV}$ at $R=15 \mathrm{fm}$ are striking. In consequence of these strong splittings there are avoided crossings between the $3 p_{1 / 2} \sigma$ and $2 p_{3 / 2} \sigma$ state at $18 \mathrm{fm}$ and the $4 p_{1 / 2} \sigma$ and $3 p_{3 / 2} \sigma$ state at $15.5 \mathrm{fm}$. At $R \sim 2700 \mathrm{fm}$ we also found a pseudocrossing between the $4 d_{3 / 2} \sigma$ and the $3 s \sigma$ state. The energy levels in rectangles I and II between 300 and $1000 \mathrm{fm}$ are presented separately on a linear scale in Figs. 1(c) and 1(d). The sudden energy change of the strongest bound states near the distance of closest approach is most impressive if energies as a function of two-center distance $R$ are drawn on a linear scale [Fig. 1 (b)]. In the region $15 \mathrm{fm} \leqslant R \leqslant 100 \mathrm{fm}$ we found the following energy changes: $330 \mathrm{keV}$ for the $1 s \sigma$ state, $208 \mathrm{keV}$ for the $2 p_{1 / 2} \sigma$ state, and $91 \mathrm{keV}$ for the $2 s \sigma$ state. In Fig. 1(c) we note especially the avoided crossings between the $4 s \sigma$ and the $4 d_{3 / 2} \sigma$ states at $400 \mathrm{fm}$ and between the 
$4 s \sigma$ and the $4 d_{5 / 2} \sigma$ states at $625 \mathrm{fm}$. The interaction of the energy levels between the $4 s \sigma$ and the $4 d_{3 / 2} \sigma$ states is much larger than that between the $4 s \sigma$ and the $4 d_{5 / 2} \sigma$ states. We found $\Delta E=0.35 \mathrm{keV}$ for the avoided crossing at $R=400 \mathrm{fm}$ and equivalently $\Delta E=0.03 \mathrm{keV}$ at $R=625 \mathrm{fm}$. The energy difference at the pseudo crossing between the $4 d_{3 / 2} \pi$ and $4 d_{5 / 2} \pi$ states at $R \sim 1100 \mathrm{fm}$ is about $1 \mathrm{keV}$. Furthermore, we remark that the $4 p_{3 / 2}{ }^{\sigma}$ state shows an energy minimum at $R=425 \mathrm{fm}$.

The behavior of electron states in Fig. 1(d) is equivalent to that discussed in Fig. 1(c). We found an avoided crossing between the $3 s_{1 / 2} \sigma$ and the $3 d_{3 / 2} \sigma$ states at $R=425 \mathrm{fm}$, with a minimum energy difference of $\Delta E=0.75 \mathrm{keV}$. A second avoided crossing appears at $R=640 \mathrm{fm}$ with $\Delta E$ $=0.05 \mathrm{keV}$. A pseudocrossing results between both $3 d \pi$ states at $R \sim 1100 \mathrm{fm}$ with $\Delta E=2.6 \mathrm{keV}$.

\section{COUPLING MATRIX ELEMENTS BETWEEN BOUND STATES}

After solving the TCD equation, we can calculate the radial and rotational matrix elements. The radial elements are conveniently evaluated by use of the relation

$$
\begin{aligned}
\left(E_{m}-E_{n}\right)\left\langle\phi_{n}\left|\frac{\partial}{\partial R}\right| \phi_{m}\right\rangle & =\left\langle\phi_{n}\left|\left[\frac{\partial}{\partial R}, H\right]\right| \phi_{m}\right. \\
& =\left\langle\phi_{n}\left|\frac{\partial V_{\Upsilon C}}{\partial R}\right| \phi_{m}\right\rangle,
\end{aligned}
$$

where $V_{\mathrm{TC}}$ is the two-center Coulomb potential and $\phi_{n}$ and $\phi_{m}$ are the corresponding orthogonal wave functions. When the two-center potential is expanded into multipoles, ${ }^{50,55,56}$ this leads for $r<\frac{1}{2} R$ to

$$
\frac{\partial V_{l}}{\partial R}=-(l+1)\left(Z_{1}+(-1)^{l} Z_{2}\right) e^{2} \frac{1}{r R}\left(\frac{2 r}{R}\right)^{l+1}
$$

and for $r \geqslant \frac{1}{2} R$ leads to

$$
\frac{\partial V_{1}}{\partial R}=l\left(Z_{1}+(-1)^{l} Z_{2}\right) e^{2} \frac{1}{r R}\left(\frac{R}{2 r}\right)^{l} .
$$

with

$$
\frac{\partial V}{\partial R}=\sum_{l=0}^{\infty} \frac{\partial V_{l}}{\partial R} P_{l}(\cos \vartheta)
$$

the total matrix element becomes for the initial $(f, g)$ and final $(F, G)$ wave function:

$$
\begin{aligned}
\left\langle\phi_{f}^{\mu}(\overrightarrow{\mathrm{r}})\left|\frac{\partial V}{\partial R}\right| \phi_{i}^{\bar{\mu}}(\overrightarrow{\mathrm{r}})\right\rangle=\delta_{\mu \bar{\mu}} \sum_{l=0}^{\infty} \sum_{\kappa, \bar{\kappa}}\left(A_{(\kappa, l, \bar{\kappa})}^{\mu} \int_{0}^{\infty} \gamma^{2} d r G_{\kappa}(r) \frac{\partial V_{l}}{\partial R} g_{\bar{\kappa}}(r)\right. \\
\left.+A_{(-\kappa, l,-\bar{\kappa})}^{\mu} \int_{0}^{\infty} r^{2} d r F_{\kappa}(r) \frac{\partial V_{l}}{\partial R} f_{\bar{\kappa}}(r)\right) .
\end{aligned}
$$

The absolute value of radial matrix elements between bound states are presented in Figs. 2 and 3 for the $\mathrm{Pb}-\mathrm{Pb}$ system and in Table I for the U-U system. The radial matrix elements $\left\langle n_{f} s \sigma|\partial / \partial R| n_{i} s \sigma\right\rangle$ have the approximately same slope and size if the difference between the main quantum numbers $n_{f}-n_{i}$ is the same, e.g., $1 s \sigma-2 s \sigma, 2 s \sigma-3 s \sigma, 3 s \sigma-4 s \sigma$ or $1 s \sigma-3 s \sigma, 2 s \sigma-4 s \sigma$. The couplings to the $1 s \sigma$ state and those to the $2 s \sigma$ state are shown separately (Figs. 2 and 3 ). The most noteworthy feature of the radial matrix elements is, however, the steep increase of their magnitude even at very small $R .^{25,50,58}$ In comparison a nonrelativistic $\partial / \partial R$ matrix element scaled to the U-U system shows a decrease at small distances $R .{ }^{58}$ The difference is due to the fact that for $Z_{1}+Z_{2}>137$ the $n s \sigma$ and $n p_{1 / 2} \sigma$ wave functions are extremely sensitive to changes in $R$, especially for small separations. This is related to the lack of a "runway" in the correlation diagram. We find that the matrix elements between the $n s \sigma$ and the $(n+k) s \sigma$ states scale as $1 /(k+1)^{2}$ to a good approximation.
Figures 4 and 5 present the $\partial / \partial R$ matrix ele ments between the low-lying odd parity states of the $\mathrm{U}-\mathrm{U}$ and $\mathrm{Pb}-\mathrm{Pb}$ quasimolecules. The sharp dis continuities in Fig. 4 occurring at $R \sim 50 \mathrm{fm}$ are due to the avoided crossing between the $2 p_{3 / 2} \sigma$ and $3 p_{1 / 2} \sigma$ levels at this separation. This influences the adiabatic states strongly, but will have only little effect in a collision (diabatic states). In comparison the results obtained in the monopole approximation [only $\dot{l}=0$ in Eqs. (22) and (23)] show a smooth behavior, thus contributing a convenient basis of diabatic molecular states. Since it is known that the discontinuities due to avoided crossings in full adiabatic twocenter calculations may often be neglected in the evaluation of dynamical processes, the monopole approximation provides an excellent starting basis for numerical calculations, being even superior to the full two-center basis at small internuclear distances due to the general diabetic character of the states.

Next we shall discuss the influence of the translation factors ${ }^{59}, 71$ on the transition strength between 


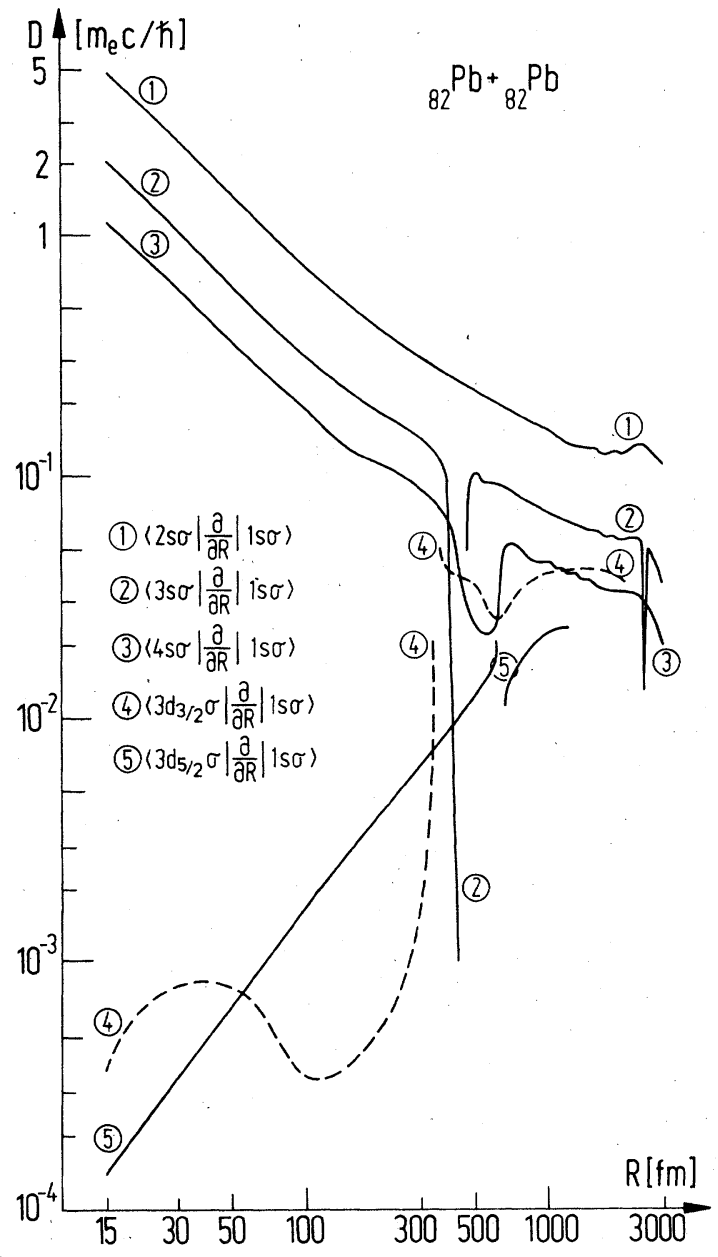

FIG. 2. Relativistic two-center calculations for the absolute value of $\partial / \partial R$ matrix elements with the $1 s \sigma$ state in the $\mathrm{Pb}-\mathrm{Pb}$ system. The discontinuities at $R$ $\sim 500 \mathrm{fm}$ result from avoided crossings.

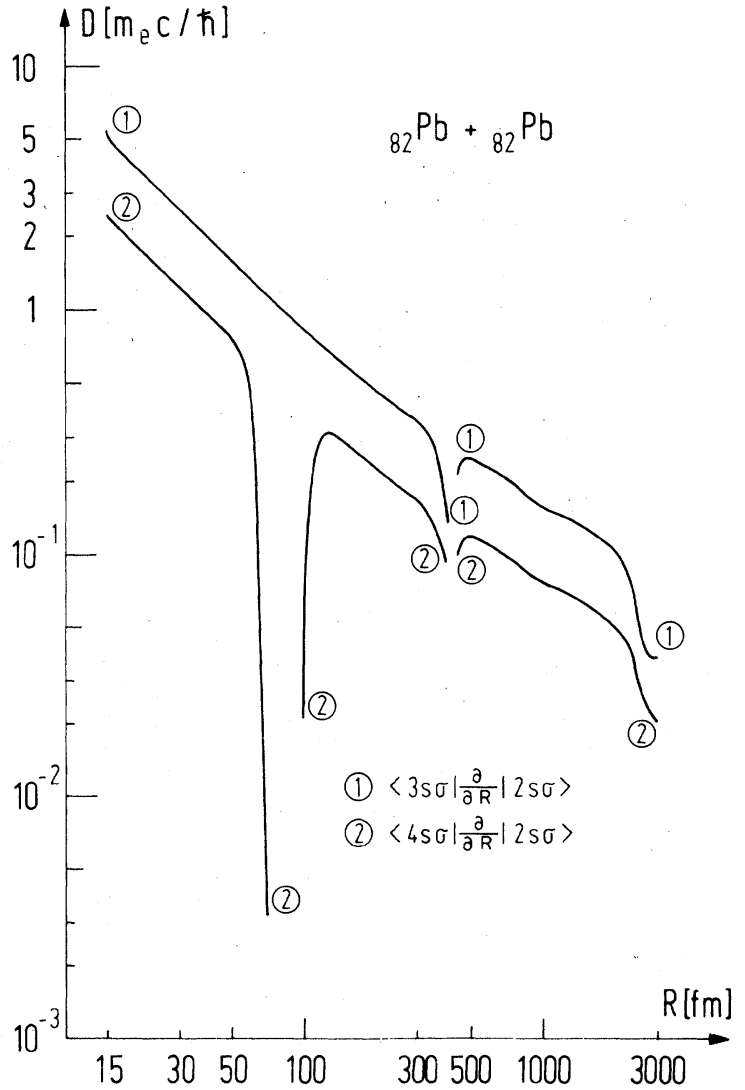

FIG. 3. Same as in Fig. 2 for the $2 s \sigma$ initial state.

bound states. In order to obtain constant occupa tion amplitudes $a_{n}(t)$ for $t \rightarrow \infty$ in dynamical calculations, it is necessary that the coupling matrix elements vanish asymptotically. The $\partial / \partial R$ matrix elements evaluated according to (24) exhibit con-

TABLE I. Relativistic two-center calculations of $\partial / \partial R$ matrix elements between $n s \sigma$ states in the U-U system as a function of the internuclear separation $R$.

\begin{tabular}{|c|c|c|c|c|c|c|}
\hline$R(\mathrm{fm})$ & $\left\langle 2 s \sigma\left|\frac{\partial}{\partial R}\right| 1 s \sigma\right\rangle$ & $\left|\left\langle 3 s \sigma\left|\frac{\partial}{\partial R}\right| 2 s \sigma\right\rangle\right|$ & $\left\langle 4 s \sigma\left|\frac{\partial}{\partial R}\right| 3 s \sigma\right\rangle$ & $\left|\left\langle 3 s \sigma\left|\frac{\partial}{\partial R}\right| 1 s \sigma\right\rangle\right|$ & $\mid\left\langle 4 s \sigma\left|\frac{\partial}{\partial R}\right| 2 s \sigma\right\rangle$ & $\mid\left\langle 4 s \sigma\left|\frac{\partial}{\partial R}\right| 1 s \sigma\right\rangle$ \\
\hline 20 & 5.6808 & 5.0676 & 5.3012 & 2.3397 & 2.2857 & 1.2831 \\
\hline 30 & 3.6754 & 3.4366 & 3.5668 & 1.5039 & 1.5658 & 0.8353 \\
\hline 50 & 2.1322 & 2.1204 & 2.1890 & 0.8802 & 0.9773 & 0.4974 \\
\hline 70 & 1.5297 & 1.5826 & 1.6316 & 0.6396 & 0.7345 & 0.3657 \\
\hline 100 & 1.0677 & 1.1438 & 1.1610 & 0.4540 & 0.5265 & 0.2589 \\
\hline 150 & 0.6857 & 0.7548 & 0.7772 & 0.2979 & 0.3552 & 0.1750 \\
\hline 200 & 0.5387 & 0.5988 & 0.6153 & 0.2378 & 0.2644 & 0.1411 \\
\hline 300 & 0.3769 & 0.4152 & 0.4121 & 0.1705 & 0.1947 & 0.1024 \\
\hline 400 & 0.2993 & 0.2979 & 0.1352 & 0.1086 & 0.0560 & 0.0477 \\
\hline 500 & 0.2582 & 0.2840 & 0.2887 & 0.1130 & 0.1361 & 0.0684 \\
\hline 700 & 0.2061 & 0.2242 & 0.2296 & 0.0939 & 0.1081 & 0.0574 \\
\hline 850 & 0.1894 & 0.2024 & 0.2067 & 0.0867 & 0.0978 & 0.0532 \\
\hline 1000 & 0.1666 & 0.1756 & 0.1793 & 0.0766 & 0.0852 & 0.0472 \\
\hline 1500 & 0.1437 & 0.1358 & 0.1365 & 0.0649 & 0.0663 & 0.0398 \\
\hline 2000 & 0.1568 & 0.0891 & 0.0959 & 0.0628 & 0.0455 & 0.0372 \\
\hline 2500 & 0.1366 & 0.0493 & 0.0457 & 0.0437 & 0.0282 & 0.0238 \\
\hline 3000 & 0.1238 & 0.0532 & 0.0437 & 0.0287 & 0.0297 & 0.0126 \\
\hline
\end{tabular}




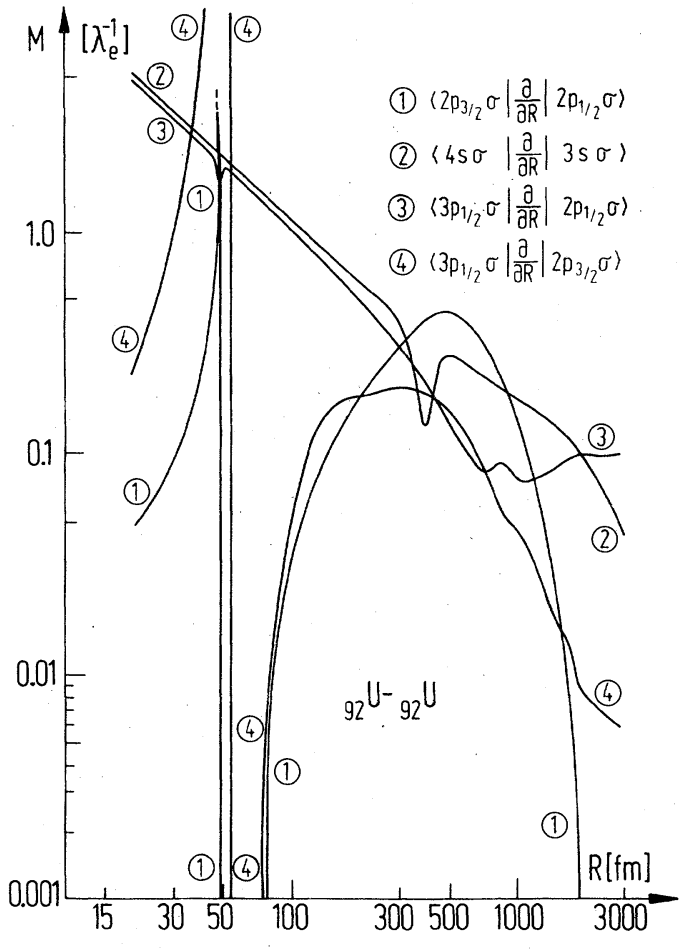

FIG.4. Absolute value of $\partial / \partial R$ matrix elements between low-lying states of the $U+U$ quasimolecule. The sharp discontinuities at $R \sim 50 \mathrm{fm}$ are due to the avoided crossing between the $2 p_{3 / 2} \sigma$ and $3 p_{1 / 2} \sigma$ levels at this separation.

stant tails which give rise to unphysical oscilla tions in the amplitudes at large separations. This behavior is known to be due to the failure of the stationary molecular states to be asymptotic solutions of the scattering equation with two atoms moving apart with velocity $\vec{v}$. If the velocity $\vec{v}$ is of constant magnitude and parallel to the $z$ axis, then we have to multiply each separated atoms wave function by a "translation" factor $\exp \left( \pm \frac{1}{2} i m_{e} \overrightarrow{\mathrm{v}} \cdot \overrightarrow{\mathrm{r}}\right)$ depending in sign on which side the wave function is centered. Note that the translation factor is only correct for large two-center distances $R$, where the straight-line trajectory is a good approximation and the electron is localized on one nucleus. To describe symmetric systems, one has first to form combinations of an even and the corresponding odd parity molecular state to describe a single separated atoms wave function and then again to take the (anti-or) symmetric combination of the boosted states:

$$
\begin{aligned}
\tilde{\phi}_{n}^{( \pm)} & =\frac{1}{2}\left(\phi_{n}^{(+)}+\phi_{n}^{(-)}\right) e^{(i / 2)_{m} \overrightarrow{\mathrm{r}}} \pm \frac{1}{2}\left(\phi_{n}^{(+)}-\phi_{n}^{(-)}\right) e^{-(i / 2)_{m} \overrightarrow{\mathrm{v}}} \\
& =\left\{\begin{array}{l}
\phi_{n}^{(+)} \cos \left(\frac{1}{2} m \overrightarrow{\mathrm{v}} \overrightarrow{\mathrm{r}}\right)+i \phi_{n}^{(-)} \sin \left(\frac{1}{2} m \overrightarrow{\mathrm{v}} \overrightarrow{\mathrm{r}}\right) \\
i \phi_{n}^{(+)} \sin \left(\frac{1}{2} m \overrightarrow{\mathrm{v}} \overrightarrow{\mathrm{r}}\right)+\phi_{n}^{(-)} \cos \left(\frac{1}{2} m \overrightarrow{\mathrm{v}} \overrightarrow{\mathrm{r}}\right) .
\end{array}\right.
\end{aligned}
$$

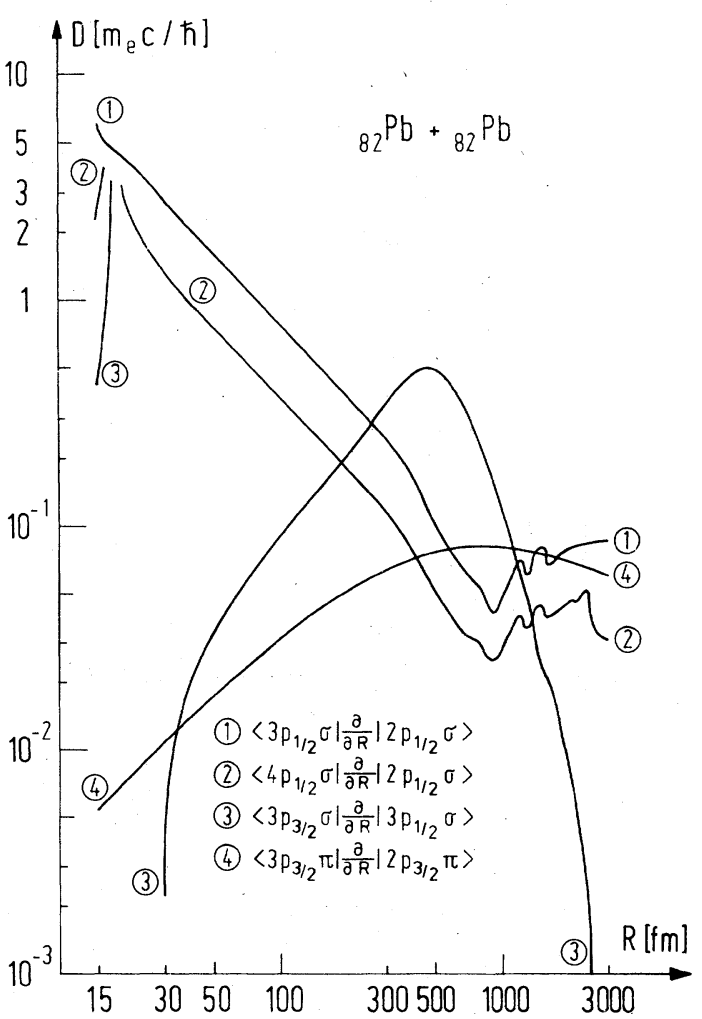

FIG. 5. Absolute value of $\partial / \partial R$ matrix elements between low-lying states of the U+U quasimolecule.

For U-U collisions at $v / c \sim 0.1$ we find that the $\sin / \cos$ factors oscillate once through $2 \pi$ in 50000 $\mathrm{fm}$, making only a small effect on the $1 s \sigma$ level which is localized within less than $1000 \mathrm{fm}$. It is therefore reasonable to include the translation factors only up to first order in the projectile velocity $v$. The matrix elements of the so-corrected wave functions (25) are in the dipole approximation $^{80}$ :

$$
\begin{aligned}
\left\langle\tilde{\phi}_{n}^{(+)}\left|\frac{\partial}{\partial R}\right| \tilde{\phi}_{m}^{(+)}\right\rangle= & \left\langle\phi_{n}^{(+)}\left|\frac{\partial}{\partial R}\right| \phi_{m}^{(+)}\right\rangle \\
& -\frac{m}{2}\left(E_{m}^{(+)}-E_{n}^{(-)}\right)\left\langle\phi_{n}^{(-)}|z| \phi_{m}^{(+)}\right\rangle .
\end{aligned}
$$

If $\phi_{n}^{(+)}=|1 s \sigma\rangle$, then $\phi_{n}^{(-)}=\left|2 p_{1 / 2} \sigma\right\rangle$, etc. $z$ is the intrinsic coordinate along the molecular axis. The matrix elements (26) vanish rapidly beyond $R$ $=2000 \mathrm{fm}$, indicating the point where the tails of the two $K$ shells just begin to touch.

In the truly molecular region $(R \lessgtr 1000 \mathrm{fm})$ the use of the full velocity $\vec{v}$ in the translation phase factor does not seem to be justified since the electron belongs to neither nucleus. Various authors $\mathrm{s}^{59}$ 


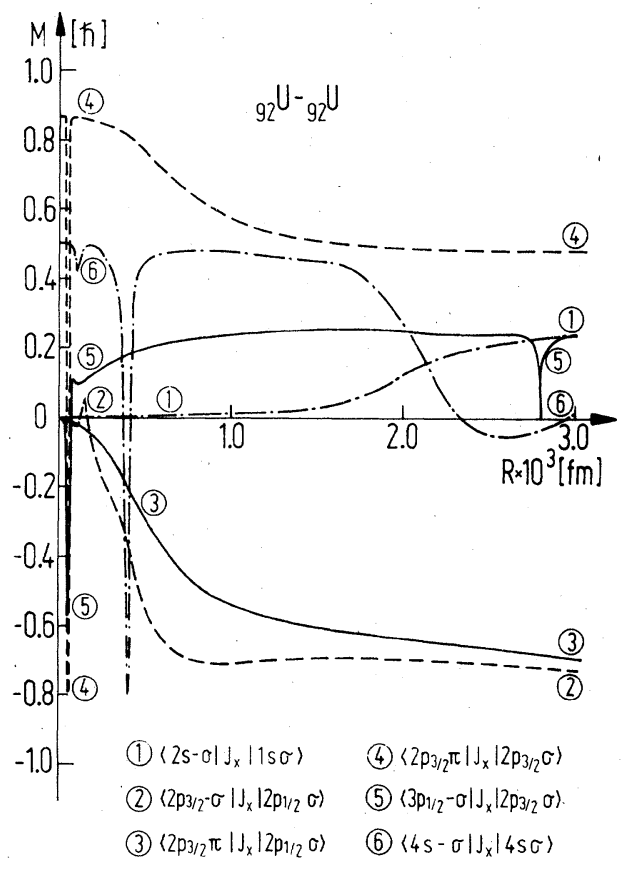

FIG. 6. Rotational-coupling matrix elements of the $\mathrm{U}+\mathrm{U}$ system. The sharp discontinuities are due to the avoided crossings between the following levels: $2 p_{3 / 2} \sigma, 3 p_{1 / 2} \sigma \cdot(R \sim 50 \mathrm{fm}) ; 4 s \sigma, 4 d \sigma(R \sim \mathrm{fm})$ and $4 s \sigma, 4 d \sigma(R \sim 400 \mathrm{fm})$.

have tried to remedy this by introduction of a weight function $f(R, \overrightarrow{\mathbf{r}})$ in front of $v$. The $\mathrm{U}-\mathrm{U}$ system is favorable in this respect, because at small $R$ the effect of the phase factor tends to become a very small correction to the matrix ele-

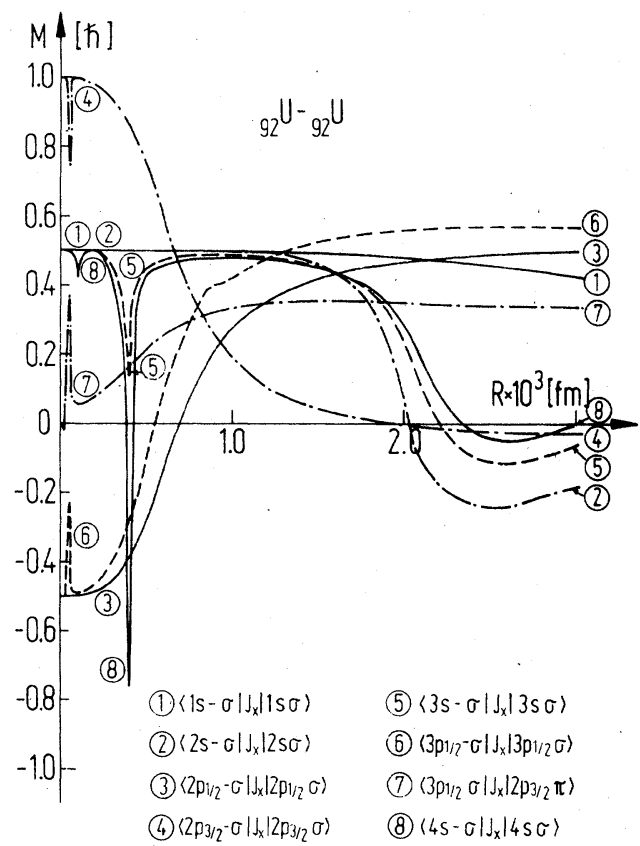

FIG. 7. Rotational-coupling matrix elements of the $\mathrm{U}+\mathrm{U}$ quasimolecule. The sharp discontinuities are due to the avoided crossings between the following levels: $2 p_{3 / 2} \sigma, 3 p_{1 / 2} \sigma(R \sim 50 \mathrm{fm}) ; 4 s \sigma, 4 d \sigma(R \sim 100 \mathrm{fm})$; $3 s \sigma, 3 d \sigma(R \sim 400 \mathrm{fm})$ and $4 s \sigma, 4 d \sigma(R \sim 400 \mathrm{fm})$.

ment. In consequence, our choice has been $f(R)$ $=1$ everywhere.

Due to the intrinsic rotation of the quasimolecule with angular velocity $\vec{\omega}=\omega \overrightarrow{\mathrm{e}}_{x}, \omega=b v(\infty) / R^{2}$, there also exist rotational matrix elements:

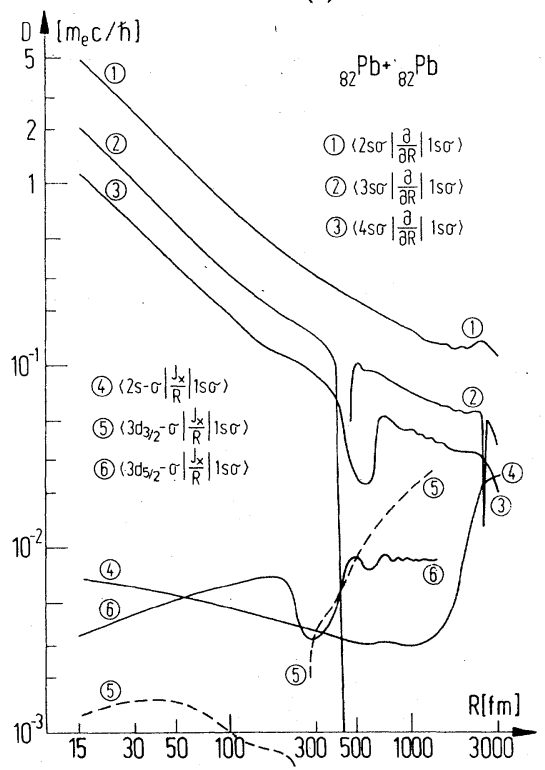

(b)

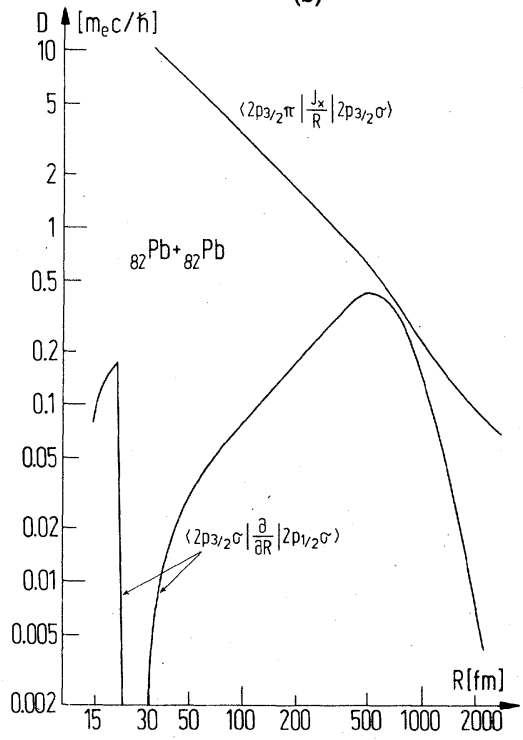

FIG. 8. Comparison between some absolute values of $\partial / \partial R$ - and $J_{x} / R$ matrix elements in the $\mathrm{Pb}-\mathrm{Pb}$ system. 


$$
\begin{aligned}
& \left\langle\phi_{f}^{\mu}(\overrightarrow{\mathrm{r}})\left|J_{x}\right| \phi_{i}^{\bar{\mu}}(\overrightarrow{\mathrm{r}})\right\rangle \\
& =\sum_{\kappa} \frac{1}{2}\left(\int_{0}^{\infty} r^{2} d r F_{\kappa}(r) f_{\kappa}(r)\left\{[(j-\bar{\mu})(j+\bar{\mu}+1)]^{1 / 2} \delta_{\mu, \bar{\mu}+1}+[(j+\bar{\mu})(j-\bar{u}+1)]^{1 / 2} \delta_{\mu, \bar{\mu}-1}\right\}\right. \\
& \left.\quad+\int_{0}^{\infty} r^{2} d r G_{\kappa}(r) g_{\kappa}(r)\left\{[(j-\bar{\mu})(j+\bar{\mu}+1)]^{1 / 2} \delta_{\mu, \bar{\mu}+1}+[(j+\bar{\mu})(j-\bar{\mu}+1)]^{1 / 2} \delta_{\mu, \bar{\mu}-1}\right\}\right) .
\end{aligned}
$$

Figures 6 and 7 show the rotational-coupling matrix elements $\left\langle J_{x}\right\rangle$ for the $U-U$ system. The sharp discontinuities at several points are due to the avoided crossing between states with the same parity and magnetic quantum number $\mu$. The spin-orbit interaction causes a splitting of the $2 p_{3 / 2} \sigma$ and the $2 p_{3 / 2} \pi$ states. In the correlation diagram Fig. 1 we see that the corresponding energy difference is small. It was shown that the relativistic states $2 p_{3 / 2} \sigma, 2 p_{3 / 2} \pi, 2 p_{1 / 2} \sigma$, and the nonrelativistic states $2 p \sigma, 2 p \pi$ are related by an angular momentum $(j \rightarrow l-s)$ recoupling scheme: The $2 p_{3 / 2} \sigma$ state becomes a $2 p \pi$-like state for large separations. That is the reason why the two matrix elements

$$
\left\langle 2 p_{3 / 2}-\sigma\left|J_{x}\right| 2 p_{1 / 2} \sigma\right\rangle,\left\langle 2 p_{3 / 2} \pi\left|J_{x}\right| 2 p_{1 / 2} \sigma\right\rangle
$$

join with increasing $R$ (see Fig. 6).

To obtain a comparison between the radial and rotational coupling we have drawn in Fig. 8 some $\partial / \partial R$ and $J_{x} / R$ matrix elements in the same units. Note that the actual rotational matrix element is less than the drawn curve, because

$$
(1 / \dot{R})|\vec{\omega} \cdot \overrightarrow{\mathrm{j}}|=\left(v_{\infty} b / \dot{R} R^{2}\right) j<j / R,
$$

where $b$ is the impact parameter and $R$ is the twocenter distance. As the most important result for the following ionization calculations we emphasize that the rotational coupling vanishes for small distances $R$. Since the coupling strength between the $n s \sigma$ and the lowest accessible states with even parity $3 d_{3 / 2} \sigma$ and $3 d_{5 / 2} \sigma$ is negligible for small $R$ we have included only $n s \sigma$ bound states for calculations of the $1 s \sigma$ vacancy production in a symmetric collision system.

\section{THE MONOPOLE APPROXIMATION}

In the calculation of direct excitation into the upper continuum $\left(E>m_{e} c^{2}\right)$ we apply the monopole approximation, where only the term with $l=0$ in the multipole expansion of the two-center potential is taken into account. In this approximation a charged spherical shell of radius $R_{n}=\frac{1}{2} R$ simulates the Coulomb potential of two nuclei separated by distance $R$.

We solve the Dirac equation with the monopole potential taking into acconnt the finite size of both nuclei, whereby binding energies and transition strengths are reduced compared with point-nuclei results. Assuming nonoverlapping homogeneously charged spheres with radius $R_{\text {nuc }}$, the $\partial / \partial R$ matrix elements between initial state $\phi_{i}$ and final state $\phi_{f}$ become

$$
\begin{aligned}
\left\langle\phi_{f}\left|\frac{\partial}{\partial R}\right| \phi_{i}\right\rangle=\frac{2\left(Z_{1}+Z_{2}\right) e^{2}}{R^{2}\left(E_{i}-E_{f}\right)} \int_{0}^{r_{+}} & \phi_{f}(R, r) \phi_{i}(R, r) \\
& \times M\left(R, r, R_{\text {nuc }}\right) r^{2} d r .
\end{aligned}
$$

Here

$$
\begin{aligned}
& M\left(R, r, R_{\text {nuc }}\right)=1 \text { for } r<r_{-} \\
& \begin{aligned}
M\left(R, r, R_{\text {nuc }}\right)=\left(1 / R_{\text {nuc }}^{3}\right)\left\{(1 / 16 r) r_{-}^{3}\left(\frac{1}{2} R+3 R_{\text {nuc }}\right)-\frac{1}{4} r_{+}^{2}\left(\frac{1}{2} R-2 R_{\text {nuc }}\right)+\frac{3}{8}\left(\frac{1}{4} R^{2}-R_{\text {nuc }}^{2}\right) r-\frac{1}{8} R r^{2}+\frac{1}{16} r^{3}\right. \\
\left.-R\left[(1 / 16 r) \frac{3}{2} r_{-}^{2}\left(\frac{1}{2} R+3 R_{\text {nuc }}\right)+(1 / 32 r) r_{-}^{3}+\frac{3}{16} R r-\frac{1}{8} r_{+}^{2}-\frac{1}{4} r_{+}\left(\frac{1}{2} R-2 R_{\text {nuc }}\right)-\frac{1}{8} r^{2}\right]\right\} \\
\text { for } r_{-}<r<r_{+}
\end{aligned}
\end{aligned}
$$

and

$$
\begin{aligned}
& r_{+}=\frac{1}{2} R+R_{\text {nuc }}, \\
& r_{-}=\frac{1}{2} R-R_{\text {nuc }} .
\end{aligned}
$$

For pointlike nuclei $\left(R_{\text {nuc }}=0\right)$ the monopole potential is

$$
V_{0}=\begin{aligned}
& -2\left(Z_{1}+Z_{2}\right) e^{2} / R \text { for } r \leqslant \frac{1}{2} R \\
& -\left(Z_{1}+Z_{2}\right) e^{2} / r \text { for } r>\frac{1}{2} R,
\end{aligned}
$$

and the factor $M$ in Eq. (28) reduces to $M=1$. As an example the finite nuclear size $\left(R_{\text {nuc }} \sim 7 \mathrm{fm}\right)$ reduces the $1 s \sigma$ binding energy in the $\mathrm{Pb}-\mathrm{Pb}$ system at $R=15 \mathrm{fm}$ by about $5 \%$. The influence on cou- 


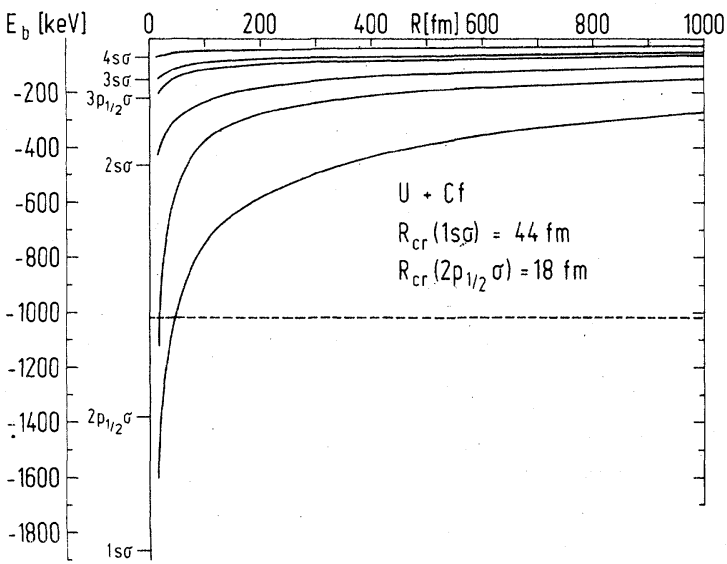

FIG. 9. Relativistic correlation diagram for the $\mathrm{U}+\mathrm{Cf}$ system calculated in the monopole approximation . The binding energies in the total united atom limit with $Z=190$ are also indicated. At the critical separation $R_{\mathrm{cr}}=44 \mathrm{fm}$ the $1 s \sigma$ state dives into the negative energy continuum.

pling matrix elements, which is much stronger, will be discussed later.

Systematic comparisons have shown that the electronic binding energy of $n s \sigma$ states in superheavy quasimolecules is reproduced correctly up to $5 \%$ accuracy by the monopole potential compared with exact two-center calculations, if the distance between the ions is not too large $(R<1000$ $\mathrm{fm})$. A detailed discussion of the validity of the monopole approximation has been published elsewhere. ${ }^{60}$ As an example we evaluated radial matrix elements between $n s \sigma$ bound states in the monopole approximation, and compared these data with the exact two-center calculations. We found good agreement within $2 \%$ for the $\mathrm{U}-\mathrm{U}$ and $\mathrm{Pb}-\mathrm{Pb}$ system in the range of $20 \mathrm{fm} \leqslant R \leqslant 400 \mathrm{fm}$. The accuracy of the monopole approximation for $\mathrm{Xe}$ $\mathrm{Pb}$ and similarly for the symmetric system ErEr is better than $6 \%$ up to $R \sim 1000 \mathrm{fm}$. The difference in the radial coupling matrix elements $\langle 2 s \sigma|\partial / \partial R| 1 s \sigma\rangle$ between the symmetric and asymmetric system is only about $5 \%$ for $R \lessgtr 500 \mathrm{fm}$, which leads to the important conclusion that the $1 s \sigma$ ionization rate in superheavy quasimolecules is a function of the total charge $Z=Z_{1}+Z_{2}$, but to a very good approximation independent of $Z_{1} / Z_{2}$, at least for asymmetries on the range $1 \geqslant Z_{L} / Z_{H}$ $\gtrsim 0.65$. As an example for a correlation diagram calculated within the monopole approximation we show in Fig. 9 the electronic binding energies as a function of two-center distance $R$ for one of the heaviest systems accessible to experimental investigation.

It is proposed to produce the superheavy electronic quasimolecule $Z=Z_{1}+Z_{2}=190$ in collisions of ${ }_{92} \mathrm{U}$ as projectile and ${ }_{98} \mathrm{Cf}$ as target. The finite nuclear sizes of projectile and target are taken into account. At a critical distance of $R_{\mathrm{cr}}=44 \mathrm{fm}$ the $1 s \sigma$ state dives into the lower continuum ( $E$ $\left.<-m_{e} c^{2}\right)$. The same happens for the $2 p_{1 / 2} \sigma$ state at $R_{\mathrm{cr}}=18 \mathrm{fm}$. For $R<R_{\mathrm{cr}}$ electronic energies

(a)

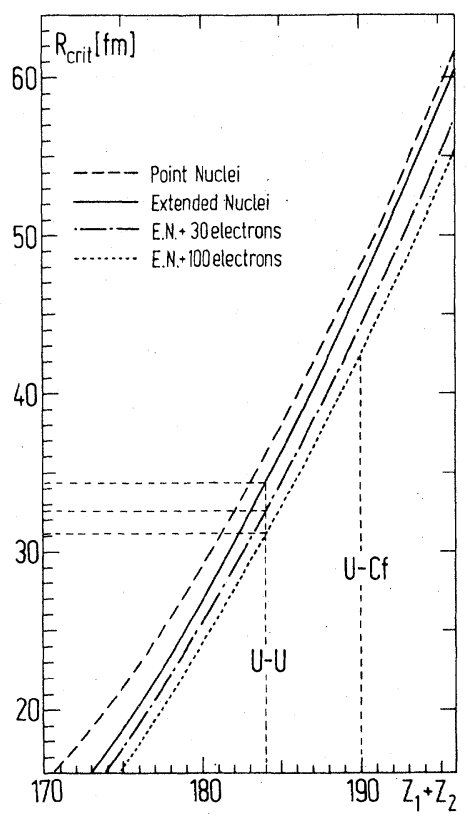

(b)

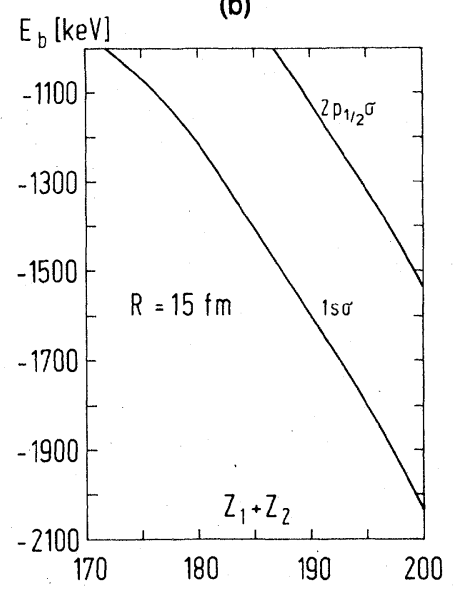

FIG. 10. (a) Critical two-center distance for the $1 s \sigma$ state as a function of total nuclear charge $Z_{1}+Z_{2}$. The corresponding values for point and extended nuclei are given separately. The screening corrections have been treated within a Thomas-Fermi model including 30 and 100 electrons, respectively. (b) Binding energies for the $1 s \sigma$ and $2 p_{1 / 2} \sigma$ electrons at a two-center distance $R=15$ $\mathrm{fm}$ as a function of total nuclear charge $Z_{1}+Z_{2}$. The finite nuclear size has been taken into account. The binding energies are determined by finding the resonance position in the negative energy continuum. 
are calculated by determination of the resonance position in the lower continuum. For $R=15 \mathrm{fm}$ we find $E_{1 s \sigma}=-1600 \mathrm{keV}, E_{2 s \sigma}=-425 \mathrm{keV}, E_{3 s \sigma}$ $=-145 \mathrm{keV}$, and $E_{2 p_{1} / 2^{\sigma}}=-1120 \mathrm{keV}$. The $2 p$ splitting is $E_{2 p_{3} / 2^{\pi}}-E_{2 p_{1 / 2}{ }^{\sigma}}=980 \mathrm{keV}$. The drastic energy change between $R=100 \mathrm{fm}$ and $R=15 \mathrm{fm}$ amounts to $850 \mathrm{keV}$ for the $1 s \sigma$ state and to 740 $\mathrm{keV}$ for the $2 p_{1 / 2} \sigma$ state, respectively. The relative change in the binding energy is therefore maximal for the $2 p_{1 / 2} \sigma$ level.

Figure 10(a) shows the critical two-center distance for the $1 s \sigma$ state as a function of the total nuclear charge in the range $173 \leqslant Z_{1}+Z_{2} \leqslant 200$ in cluding the influence of finite nuclear size, and the screening of 30 (100) residual electrons in the Thomas-Fermi approximation. Meanwhile an even more elaborated calculation of the influence of the electron-electron interaction on the critical internuclear separation has been presented in Ref. 87 .

To a good approximation the $1 s \sigma$ binding energy according to potential $(30)$ can be scaled by ${ }^{60}$

$$
E(Z, R)=E_{i}(15 \mathrm{fm} / R)^{\gamma} i e^{\alpha\left(z-Z_{i}\right)},
$$

with

$$
\alpha=\frac{1}{32} \ln \left[\frac{E_{i+1}}{E_{i}}\left(\frac{15 \mathrm{fm}}{R}\right)^{\gamma_{i+1}-\gamma_{i}}\right] .
$$

$i=1$ for $Z_{1} \leqslant Z \leqslant Z_{2}$ and $i=2$ for $Z_{2} \leqslant Z \leqslant 200$ with $Z_{1}=136, Z_{2}=168$. The parameters $E_{i}$ and $\gamma_{i}$ are given by $E_{1}=-381 \mathrm{keV}, E_{2}=-930 \mathrm{keV}, E_{3}$ $=-2280 \mathrm{keV}$, and $\gamma_{1}=0.1162, \gamma_{2}=0.2854, \gamma_{3}$ $=0.4899$. Equivalent parameters are also obtained $^{60}$ for the monopole potential with respect to finite nuclear size and electron screening.

Before we discuss the qualitative behavior of coupling matrix elements between bound states and continuum states let us briefly review the calculation of relativistic continuum wave functions in the monopole approximation.

\section{RELATIVISTIC CONTINUUM WAVE FUNCTIONS}

The relativistic continuum wave functions for a spherically symmetric potential $V(r)$ are given by the solutions of the radial Dirac equation ${ }^{61}(\hbar=c$ $=1$ ):

$$
\begin{aligned}
& \frac{d G}{d r}=-\frac{\kappa}{r} G+(E+m-V) F, \\
& \frac{d F}{d r}=-(E-m-V) G+\frac{\kappa}{r} F .
\end{aligned}
$$

Positive energies $\left(E>m_{e}\right)$ describe electron states whereas negative energies $\left(E<-m_{e}\right)$ describe positron states.

We have solved the two coupled equations of first order numerically with Hamming's modified pre- dictor-corrector method. ${ }^{57}$ This procedure generates the wave function at arbitrary points up to a normalization constant from four initial values for $G=g \cdot r=u_{1}$ and $F=f \cdot r=u_{2}$ at the origin, which must be known very accurately. We normalize the continuum wave functions to $\delta$ functions in the energy variable:

$$
\int_{0}^{\infty}\left(G_{E} G_{E^{\prime}}+F_{E} F_{E^{\prime}}\right) d r=\delta\left(E-E^{\prime}\right) .
$$

For the cutoff Coulomb potential $\left(\alpha=e^{2} / \hbar c\right)$

$$
\begin{aligned}
& V=-V_{0}=-\frac{Z \alpha}{d} \text { for } r \leqslant d, \\
& V=-Z \alpha / r \text { for } r>d,
\end{aligned}
$$

the regular solutions of the radial equations (33) can be given analytically. With $S_{\kappa}=\kappa /|\kappa|$ and $p^{2}=\left(E+V_{0}\right)^{2}-m_{e}^{2}$, it follows for $r \leqslant d^{61}$ :

$$
\begin{aligned}
& G=a_{0} r j_{l}(p r), \\
& F=a_{0} S_{k}\left[p r /\left(E+V_{0}+m_{e}\right)\right] j_{\bar{l}}(p \cdot r),
\end{aligned}
$$

with $\vec{l}=l-S_{k}$. The relations are valid also for $V_{0}=0$ and hence include the solution of the Dirac equation for free spin $1 / 2$ particles. In general the positron and electron radial wave functions

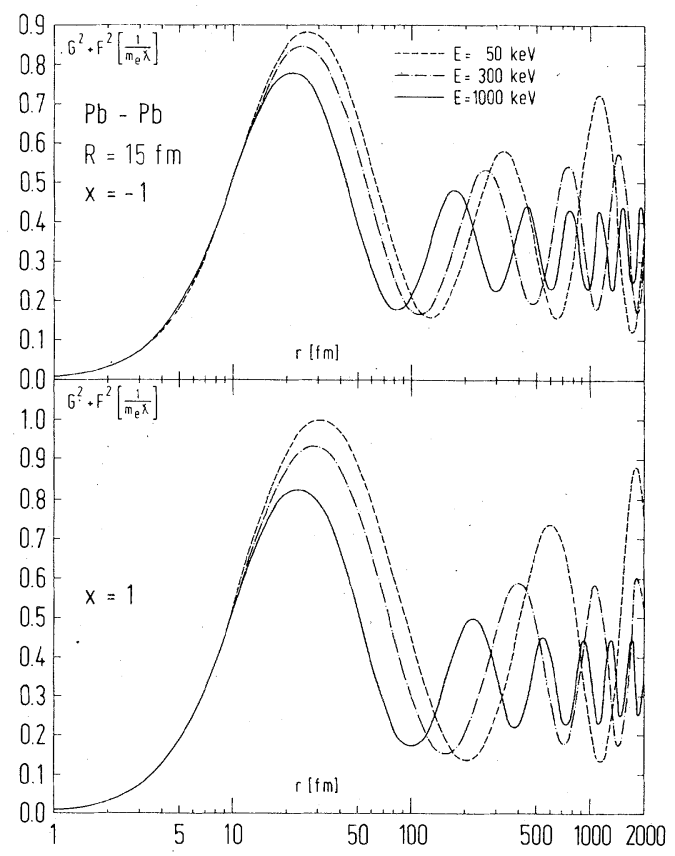

FIG. 11. Radial density distributions $|\psi r|^{2}=G^{2}+F^{2}$ of relativistic continuum wave functions with $\kappa=-1$ and $\kappa=+1$. Electron states with kinetic energy $E=50 \mathrm{keV}$, $300 \mathrm{keV}$, and $1000 \mathrm{keV}$ in the $\mathrm{Pb}-\mathrm{Pb}$ quasimolecule at a separation $R=15 \mathrm{fm}$ are considered. No difference between the wave functions is found for $r<20 \mathrm{fm}$. 
satisfy the relations

$$
\begin{aligned}
& G\left(E-V_{0}, \kappa\right)=F\left(-E+V_{0},-\kappa\right), \\
& F\left(E+V_{0}, \kappa\right)=G\left(-E-V_{0},-\kappa\right) .
\end{aligned}
$$

Solutions (36) are valid only outside the "gap" $-m_{e}-V(r) \leqslant E \leqslant+m_{e}-V(r)$. In superheavy sys tems this gap is situated at energies $E<-10 \mathrm{MeV}$, and has no relevance for ionization nor for $e^{+}, e^{-}$ pair creation in collisions of very heavy ions.

For the harmonic oscillator potential

$$
\begin{aligned}
& V(r)=-(Z \alpha / 2 d)\left(3-r^{2} / d^{2}\right) \text { for } r \leqslant d, \\
& V(r)=-Z \alpha / r \text { for } r>d,
\end{aligned}
$$

the solutions for $G$ and $F$ for $r \leqslant d$ can be given in terms of a series expansion. For $\kappa=k=|\kappa|$ we can write ${ }^{61}$

$G=\left(\frac{r}{d}\right)^{k+1} \sum_{n=0}^{\infty} a_{n}\left(\frac{r}{d}\right)^{2 n}, F=\left(\frac{r}{d}\right)^{k} \sum_{n=0}^{\infty} b_{n}\left(\frac{r}{d}\right)^{2 n}$.

$a_{n}$ and $b_{n}$ follow from the comparison of coeffi cients, if one inserts ansatz (39) in Eq. (33):

$$
\begin{aligned}
& b_{0}=(2 k+1) a_{0} /\left[d\left(E+m_{e}\right)+\frac{3}{2} Z \alpha\right], \\
& b_{1}=-\left(\frac{1}{2} d\left(E-m_{e}\right)+\frac{3}{4} Z \alpha\right) a_{0} \\
& a_{1}=\left\{\left[d\left(E+m_{e}\right)+\frac{3}{2} Z \alpha\right] b_{1}-\frac{1}{2} Z \alpha b_{0}\right\} /(2 k+3) ;
\end{aligned}
$$

for $n>1$ we have

$$
\begin{aligned}
& (2 k+2 n+1) a_{n}=\left[d\left(E+m_{e}\right)+\frac{3}{2} Z \alpha\right] b_{n}-\frac{1}{2} Z \alpha b_{n-1}, \\
& 2(n+1) b_{n+1}=-\left[d\left(E-m_{e}\right)+\frac{3}{2} Z \alpha\right] a_{n}+\frac{1}{2} Z \alpha a_{n-1} .
\end{aligned}
$$

For $\kappa=-k, G$ and $F$ as well as the sign of $E$ and $Z$ must be exchanged.

The Dirac radial functions are now determined uniquely up to the normalization constant $a_{0}$ in Eqs. (36) and (40). Two different procedures have been applied in order to extract the normalization constant $a_{0}$ from the large $-r$ behavior of the wave functions.

(i) For the potentials (35) and (38) the continuum wave functions can be represented in analytical form. ${ }^{10}$ A comparison of these analytical wave functions with values, obtained by using the numerical integration procedure at one certain point $r_{0}$, uniquely determines $a_{0}$. However, the explicit calculation of the analytical wave functions at all points $r$ cannot be recommended because of large computing time and accuracy problems for large arguments $p r$.

(ii) The asymptotic solutions for $r \rightarrow \infty$ of the radial equations (33) normalized to (34) are

$G=F_{1} \cos [p r+\delta(r)], \quad F=F_{2} \sin [p r+\delta(r)]$,

$$
\text { with } \begin{aligned}
p^{2} & =E^{2}-m_{e}^{2} \text { and } \\
F_{1} & =\left(E+m_{e}\right)^{1 / 2}(\pi p)^{-1 / 2} \text { for } E>m_{e}, \\
F_{1} & \doteq\left(-E-m_{e}\right)^{1 / 2}(\pi p)^{-1 / 2} \text { for } E<-m_{e} ; \\
F_{2} & =-\left(E-m_{e}\right)^{1 / 2}(\pi p)^{-1 / 2} \text { for } E>m_{e}, \\
F_{2} & =\left(-E+m_{e}\right)^{1 / 2}(\pi p)^{-1 / 2} \text { for } E<-m_{e} .
\end{aligned}
$$

Using the unnormalized wave functions calculated numerically up to the asymptotic region, we obtain

$$
a_{0}=\left(G_{\text {num }}^{2} / F_{1}^{2}+F_{\text {num }}^{2} / F_{2}^{2}\right)^{-1 / 2} .
$$

In Fig. 11 we show the radial density distribution $F^{2}+G^{2}$ for continuum electrons with kinetic energies of $50 \mathrm{keV}, 300 \mathrm{keV}$, and $1 \mathrm{MeV}$ for the $\mathrm{Pb}$ $\mathrm{Pb}$ quasimolecule separated by $R=15 \mathrm{fm}$. The $s(\kappa=-1)$-and $p(\kappa=1)$-wave contributions are calculated taking into account the finite nuclear size of the $\mathrm{Pb}$ nuclei.

By finding the resonance position of continuum wave functions in the negative energy continuum, we also can determine binding energies in overcritical fields. In Fig. $10(\mathrm{~b})$ the $1 s \sigma$ and $2 p_{1 / 2} \sigma$ binding energies are displayed as a function of the total nuclear charge $Z=Z_{1}+Z_{2}$ for a two-center distance $R=15 \mathrm{fm}$. For this separation (just before both nuclei touch) the $1 s \sigma$ binding energy is larger than $2 \mathrm{MeV}$ for $Z=200$.

\section{COUPLING STRENGTH BETWEEN BOUND AND CONTINUUM STATES}

The overall agreement between results obtained within the monopole approximation and exact twocenter calculations in the case of bound levels makes it possible to evaluate the matrix elements to continuum states without actually computing relativistic two-center continuum wave functions. However, this restricts our considerations to radial coupling.

For the $\mathrm{Pb}-\mathrm{Pb}$ system the numerically calculated matrix element $D_{E}=\left\langle\phi_{E}|\partial / \partial R| \phi_{b s}\right\rangle$ between the $1 s \sigma$ state and a $s \sigma$ continuum state with total energy $E\left(m_{e}=1\right)$ are displayed in Fig. 12 versus twocenter distance $R$. Qualitatively, they show the same behavior as the bound-bound state couplings, especially a rapid falloff at larger separations. The dashed lines denote the values obtained for the point nuclei. At $R=14 \mathrm{fm}$ the finite nuclear size reduces $D_{E}$ by about $30 \%$, and its influence decreases for growing $R$ and can be neglected for $R>50 \mathrm{fm}$. However, since the radial matrix elements enter quadratically into the ionization probabilities, the finite nuclear size considerably modifies the vacancy production rate. ${ }^{42}$

The dependence of the transition strength at fixed $R=14 \mathrm{fm}$ on continuum energy $E$ can be taken from Fig. 13. As initial states the $1 s \sigma, 2 p_{1 / 2} \sigma$, 


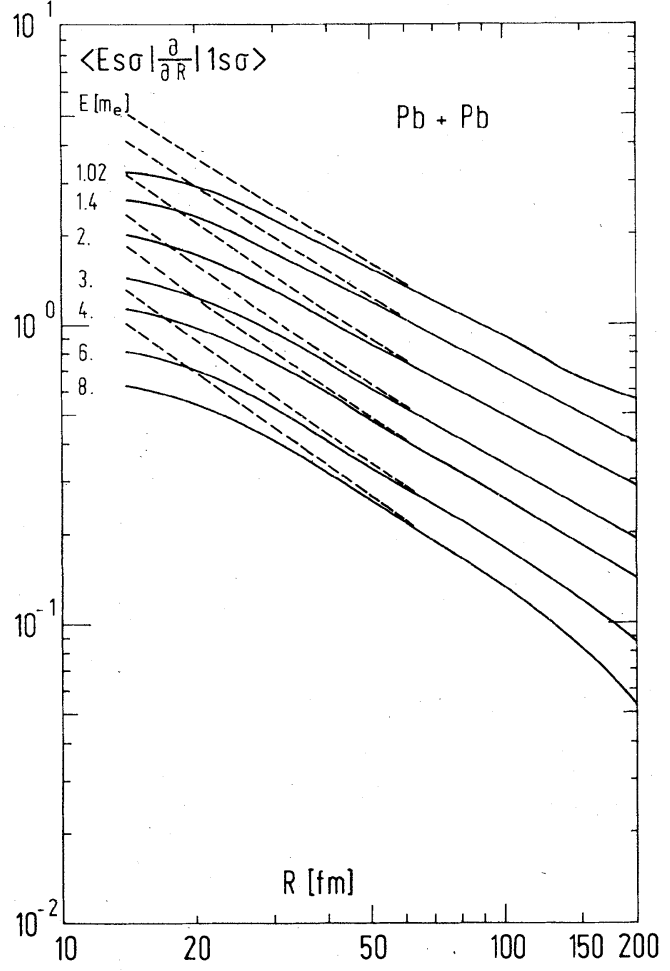

FIG. 12. Absolute value of $\partial / \partial R$ matrix elements in units $\hbar=c=m_{e}=1$ between the $1 s \sigma$ state and several continuum states with total energy $E$ as a function of two-center distance $R$ in $\mathrm{fm}$. Point-nuclei results (dashed lines) and extended nuclei results (solid lines) are shown separately for the $\mathrm{Pb}-\mathrm{Pb}$ quasimolecule.

$2 s \sigma$, and $3 s \sigma$ states are considered. For low final-state energy the matrix elements of the higher bound states dominate, whereas for $E>m_{e} c^{2}$ the $1 s \sigma$ ionization becomes more and more important. Even for very high energies the $1 s \sigma$ and $2 p_{1 / 2} \sigma$ transition strengths to the continuum stay comparable. This leads us to the important conclusion that a spectroscopy of high-energy con-

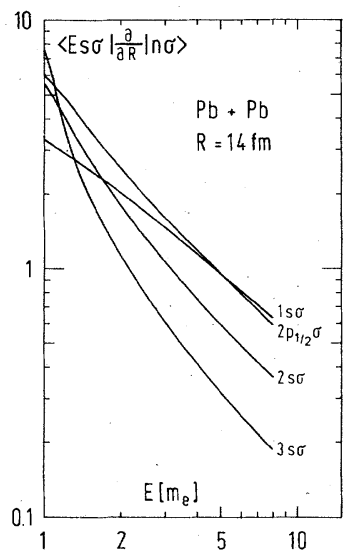

FIG. 13. Dependence on continuum energy of the absolute value of $\partial / \partial R$ matrix elements in units of $\pi=c$ $=m_{e}=1$. As initial states in the $\mathrm{Pb}+\mathrm{Pb}$ quasimolecule at an internuclear separation $R=14 \mathrm{fm}$ the $1 s \sigma$, $2 p_{1 / 2} \sigma, 2 s \sigma$, and $3 s \sigma$ states are considered.
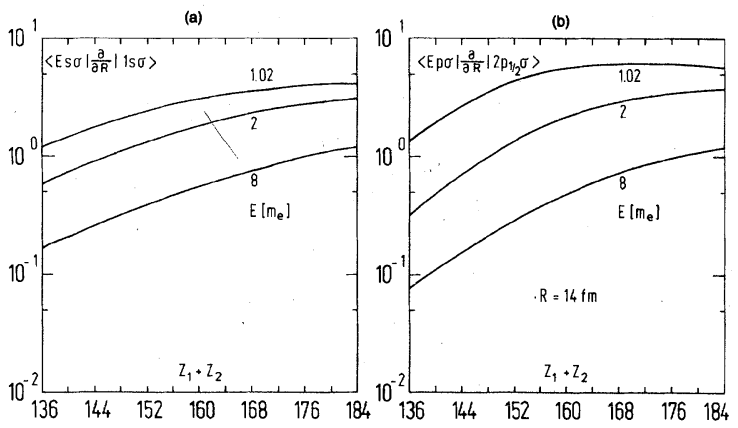

FIG. 14. Dependence on total nuclear charge $Z_{1}+Z_{2}$ of the absolute value of $\partial / \partial R$ matrix elements to the continuum in units of $\hbar=c=m_{e}=1$. The continuum states with total energy $E=1.02,2$, and $8 m_{e}$ at the two-center distance $R=14 \mathrm{fm}$ are considered.

tinuum electrons ( $\delta$ electrons) for $Z \gtrsim 164$ does not allow us to separate information of binding ener gies or form factors out of the quasiatomic $K$ shell in symmetric collisions. Because of the smaller Fourier frequencies necessary for ionization, the $2 p_{1 / 2} \sigma$ excitation always produces the dominant contribution.

In Fig. $14 D_{E}$ is plotted for the initial states $1 s \sigma$ and $2 p_{1 / 2} \sigma$ as a function of the total nuclear charge $Z=Z_{1}+Z_{2}$. For $148 \leqslant Z \leqslant 172$ we find a linear increase leveling off to a constant for $Z>180$. Finally we mention that for pointlike nuclei an overall parametrization of the radial matrix elements is achieved by a power law $\left(Z^{\prime}=184-Z\right)^{25,62}$ :

$$
\left\langle E s \sigma\left|\frac{\partial}{\partial R}\right| 1 s \sigma\right\rangle=d_{0}(Z) \frac{\left(m_{e} c^{2} / E\right)^{\gamma(z) / 2}}{R},
$$

with

$$
\begin{aligned}
& \gamma(Z)=2.08-0.0024(Z-140), \\
& d_{0}(Z)=\left[D / 4 \pi^{2} m_{e} c^{2}\right]^{1 / 2},
\end{aligned}
$$

and

$$
D(Z)=1.2+0.054(Z-158)-0.000015(Z-158)^{3} .
$$

\section{EXCITATION TO THE CONTINUUM}

In collisions of very heavy ions the strongly bound electrons can be excited directly to the continuum. We investigated this process in the framework of time-dependent perturbation theory according to Eqs. (11) and (12). ${ }^{25-27,50,58,62}$ Effects of rotational coupling in general must be treated in the strong coupling limit and therefore cannot be considered in this context. In Fig. 15 the $1 s \sigma$ ionization probability is displayed as a function of the two-center distance $R$ for a central $\mathrm{U}+\mathrm{Cf}$ collision $(b=0)$ with $R_{\min }=15 \mathrm{fm}$. The probability is integrated over all final-state continuum energies 


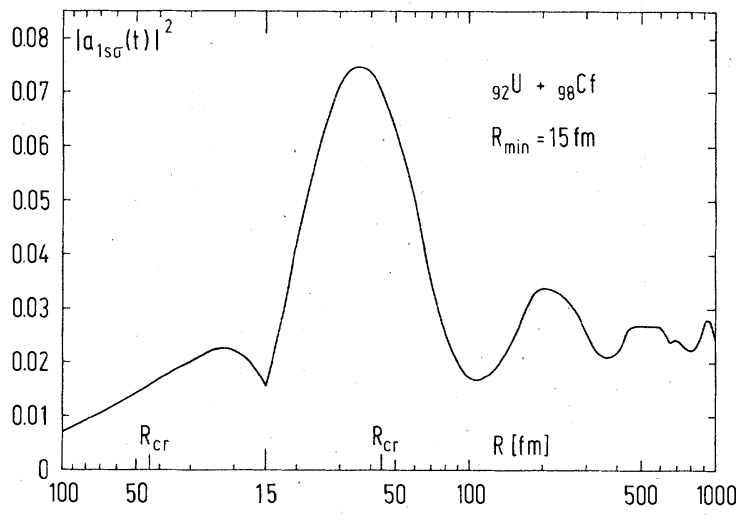

FIG. 15. Number of created $1 s \sigma$ vacancies during a head-on collision $(b=0)$ of $U$ on Cf. $R_{c r}$ denotes the two-center distance where the $1 s \sigma$ state dives into the negative energy continuum.

and the influence of the finite nuclear size has been included. The critical distance at which the quasimolecular $K$ shell dives into the negative energy continuum amounts to $R_{\text {cr }}=44 \mathrm{fm}$ for the UCf system.

The transition probability to the continuum reaches a first maximum on the incoming branch of the Coulomb trajectory with $\sum|a(R=22 \mathrm{fm})|^{2}$ $=0.023$. Due to the stronger binding for smaller separations $R$ and the lower ion velocity $v$ in the range of minimum approach, this probability is then reduced again. Near the distance of closest approach $v$ changes strongly in magnitude as well as in sign. Therefore the electrons can no longer follow the nuclear motion adiabatically. In consequence the ionization rate increases drastically and reaches a maximum at $R=36 \mathrm{fm}$ with $\sum|a(t)|_{\max }^{2}=0.075$. The average ionization probability for the diving period of the $1 s \sigma$ level is $\left|a\left(R<R_{\text {cr }}\right)\right|_{\text {av }}^{2} \sim 0.05$. There are numerous oscillations in the vacancy production rate on the outgoing
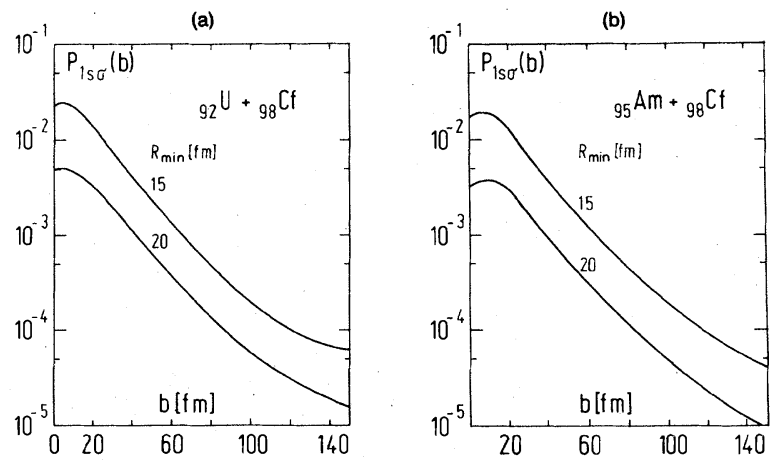

FIG. 16. Impact-parameter dependence of $1 s \sigma$ ionization to the continuum $\left(E>m_{e} c^{2}\right)$ in the superheavy quasimolecules $\mathrm{U}+\mathrm{Cf}$ and $\mathrm{Am}+\mathrm{Cf}$ at two different bombarding energies. (a)

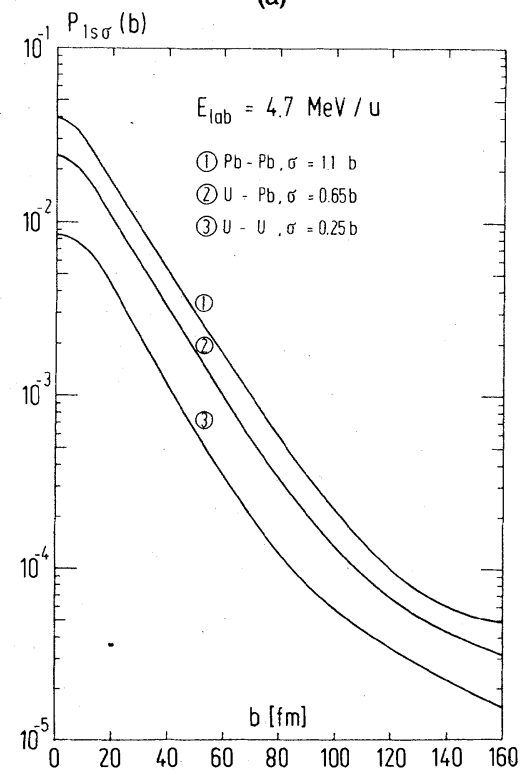

(b)

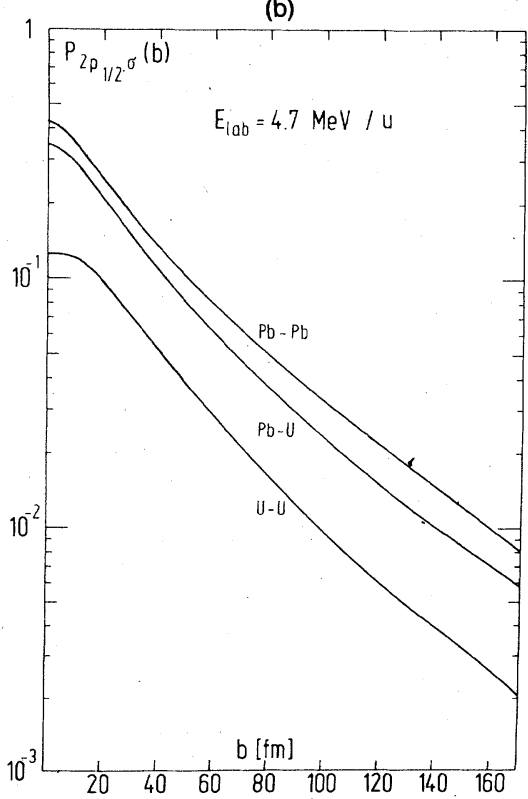

FIG. 17. Impact-parameter dependence of direct $1 s \sigma$ and $2 p_{1 / 2} \sigma$ excitation to the continuum in $\mathrm{Pb}-\mathrm{Pb}, \mathrm{Pb}-\mathrm{U}$, and U-U collisions at $E_{\text {lab }}=4.7 \mathrm{MeV} / \mathrm{u}$.

branch of the trajectory. Especially for large $R$ the integration over final states with different phase relations leads to irregular frequencies and amplitudes. The asymptotically $(t \rightarrow \infty)$ observable $K$-shell ionization probability is $P(b=0)$ $=\sum|a(t \rightarrow \infty)|^{2}=0.025$. Due to the strong relativ istic effects in the electronic levels of superheavy quasimolecules, this result is by several orders of magnitude larger than nonrelativistic extrapola- 
tions had predicted, ${ }^{63}$ but of the same order of magnitude as estimated by Burch et al. ${ }^{64}$

Upon variation of the impact parameter we find that $P(b)$ has a maximum for $P_{1 s \sigma}(b)$ at $b \sim 5-10 \mathrm{fm}$ for the systems $\mathrm{U}+\mathrm{Cf}$ and $\mathrm{Am}+\mathrm{Cf}^{65}$ (Fig. 16). In spite of the higher Fourier frequencies available in central collision $(b=0)$, the ionization probability is a little bit smaller there. This is caused by the stronger binding at smaller separation $R \sim R_{\text {min }}$ and by the reduction of the coupling strength due to the finite nuclear size. For $b>20 \mathrm{fm}$ the vacancy production probability via radial coupling to the positive continuum decreases drastically.

In Fig. 17 we compare the direct ionization of the $1 s \sigma$ and the $2 p_{1 / 2} \sigma$ level for the collisions $\mathrm{Pb}-$ $\mathrm{Pb}, \mathrm{Pb}-\mathrm{U}$, and $\mathrm{U}-\mathrm{U}$ at $E_{\mathrm{lab}}=4.7 \mathrm{MeV} / \mathrm{u}$. First of all we observe that the ionization decreases with increasing total nuclear charge due to increased binding. Furthermore, the decrease of $P(b)$ as function of impact parameter is much weaker for the $2 p_{1 / 2} \sigma$ state than for the stronger bound $1 s \sigma$ state. Here, in contrast to the results shown before, projectile and target nuclei are assumed to be pointlike. For central collisions the $2 p_{1 / 2} \sigma$ ionization is about an order of magnitude larger than that of the 1 s $\sigma$ level. We stress, however, that the impact-parameter dependence of the $2 p_{1 / 2} \sigma$ vacancy creation may be modified consider ably by the strong rotational coupling between the substates of the quasimolecular $L$ shell $^{41}$ (see Sec. $\mathrm{XI})$.

In Fig. 18 we present the impact-parameter dependence of $1 s \sigma$ ionization for the systems $\mathrm{Xe}+\mathrm{U}$ and $\mathrm{Xe}+\mathrm{Pb}$ with total charge of $Z=146$ and 136 , respectively, at various bombarding energies. Contrary to the heavier systems like $\mathrm{U}+\mathbf{C f}$, here the vacancy production rate for $b=0$ grows with increasing $Z$. As a function of $Z$ also the impactparameter dependence $P(b)$ becomes steeper, but the falloff is still much weaker than in systems with $Z_{p}+Z_{t}>160$.

The main result of this paragraph is that these results predict ionization probabilities of several percent for head-on collisions caused by the relativistic effects in the bound and continuum states. These theoretical predictions have meanwhile been verified by experiment. . $^{36-39,75,82,85}$

In Fig. 19 we compare our numerically calculated values for $P(b)$ with coincidence data of the experimental atomic physics group at GSI. ${ }^{74} \mathrm{Col}-$ lisions with $\mathrm{Pb}$ as projectile on several targets at $E_{\text {lab }}=4.7 \mathrm{MeV} / \mathrm{u}$ are investigated for different impact parameters, where the $1 s \sigma$ ionization probability was measured in coincidence with the scattered ions. Good agreement between theory and experiment is found in the slope of the exponential falloff but in general the experimental data are by

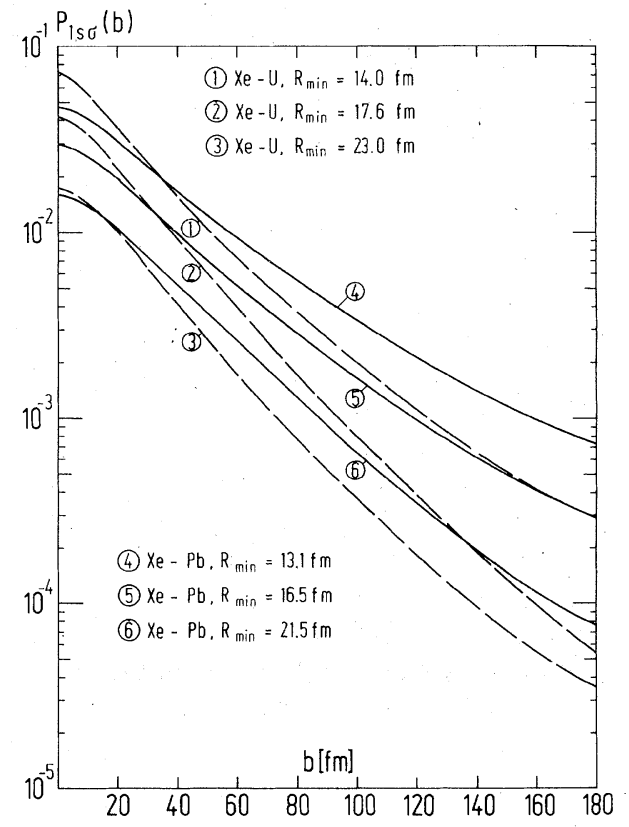

FIG. 18. Impact-parameter dependence of direct $1 s \sigma$ ionization in $\mathrm{Xe}-\mathrm{Pb}$ and $\mathrm{Xe}-\mathrm{U}$ collisions for different bombarding energies.

a factor 2 or 3 larger than the theoretical results.

We emphasize that in the calculations only transitions to the continuum but no couplings to vacant bound states are considered. ${ }^{26,83}$ Also compared with the experimental data of Greenberg et $a l .{ }^{36}$ and Anholt and Meyerhof ${ }^{39}$ for the $1 s \sigma$ ionization, our corresponding theoretical values are too small by

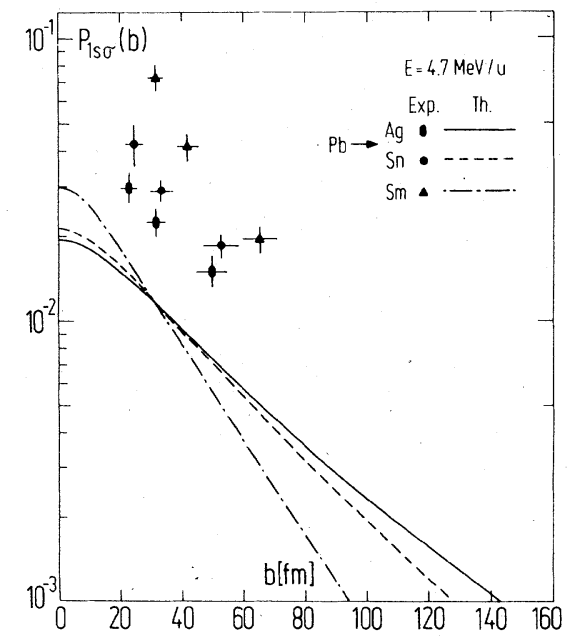

FIG. 19. Impact-parameter dependence of $1 s \sigma$ ionization in collisions with $\mathrm{Pb}$ as projectile on various targets. For comparison we show the corresponding experimental data of Ref. 74. 
about a factor of 2 or 3 , which possibly can be explained by the neglect of vacant high-lying bound states in the calculation of the ionization amplitude. In summary, the main feature of the experimental data are the large ionization probabilities of several percent and the strong falloff with increasing impact parameter. ${ }^{50,58}$

In Fig. 20 the ion energy dependence of the $1 s \sigma$ ionization is displayed for the total charges $Z=136$ and $Z=164$. Altogether the curves for $Z=164$ show a steeper dependence on $E_{\text {lab }}$ as those for $Z$ $=136$. Due to a relatively flat falloff of $P(b)$, the total ionization cross section is always larger for the lighter system independent of ion energy, despite larger vacancy-creation rates for the heavier system in central collisions at high impact energies. At large projectile energies the energy dependence of ionization scales according to a power law. This law, however, does not cover, and cannot be extended to, the whole range of projectile energy shown in the figure.

The most important result of our investigations is the possiblility to utilize the impact-parameter dependence of ionization to perform a spectroscopy of the quasimolecular states during the collision. ${ }^{26,62}$ For example, the increase of the $1 s \sigma$ ionization probability in head-on collisions up to $Z=164$ is caused by the relativistic effects in the coupling matrix elements. Beyond 164 the $1 s \sigma$

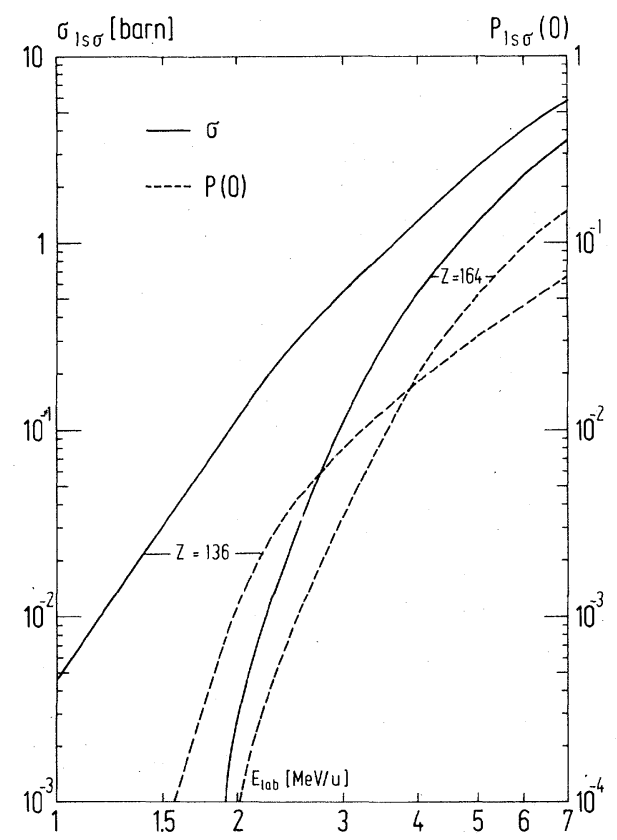

FIG. 20. Total cross section and number of created vacancies in head-on collisions $(b=0)$ for direct $1 s \sigma$ ionization as a function of the bombarding energy. The superheavy quasimolecules $Z=136$ and $Z=164$ are considered. binding energy increases rapidly as a function of $Z$, so that much higher Fourier frequencies for ionization are needed. These frequencies are scarcely available in collisions below the Coulomb barrier. In consequence the number of created vacancies per collision decreases again for $Z$ $>164$. A measurement of this behavior would be the first experimental confirmation of the strong binding of the $1 s \sigma$ electron for $Z>170$ and the diving into the negative energy continuum, as the oretically predicted. ${ }^{4,10-12,67}$

The possibility to perform a spectroscopy becomes more transparent in an analytical model of $1 s \sigma$ ionization. ${ }^{62}$ According to this model

$$
P(b)=D(Z) \exp \left[-E_{b}\left(R_{\min }\right) / \Gamma\right],
$$

with

$$
\begin{aligned}
& \Gamma(b)=\frac{\hbar v_{\infty}}{4 R_{\min }(b)}\left(2-\frac{Z_{1} Z_{2} e^{2}}{R_{\min }(b) E_{\mathrm{c} . \mathrm{m} .}}\right)^{1 / 2} . \\
& D(Z)=4.3 \exp \left(-Z^{\prime} / 12.9\right)\left[1+\exp \left(-Z^{\prime} / 7.7\right)\right]^{-1},
\end{aligned}
$$

$Z^{\prime}=184-Z$ and $v_{\infty}$ is the incident ion velocity. $R_{\text {min }}(b)$ denotes the minimum two-center distance on a given Coulomb trajectory and $E_{\text {c.m. }}$. the kinetic ion energy in the center of mass frame. According to formula (49) the impact-parameter dependence of ionization essentially shows an exponential behavior. The main feature of formula (49) is the one-to-one correspondence between the binding energy $E_{b}\left(R_{\min }\right)$ of the $1 s \sigma$ level at the dis tance of closest approach and the impact-parameter dependence of the ionization probability $P(b)$, which can be measured experimentally. For the calculation of binding energies formula (29) can be used.

Before we discuss some aspects of vacancy creation caused by radial and rotational couplings be tween bound states we briefly comment on a background contribution to inner-shell ionization. In duced by nuclear Coulomb excitation in collisions of very heavy ions, internal conversion may take place. This process is particularly important for deformed heavy nuclei like ${ }^{238} \mathrm{U}$, whereas its contribution for ${ }^{208} \mathrm{~Pb}$ is completely negligible. This is caused by rather weakly populated high-lying excited states of the ${ }^{208} \mathrm{~Pb}$ nucleus $\left(3^{-}\right.$at 2.615 $\mathrm{MeV}$ and $2^{+}$at $4.086 \mathrm{MeV}$ ) and by a small conversion coefficient $\left(\alpha<10^{-3}\right)$ for the corresponding transitions. In ${ }^{238} \mathrm{U}$, on the other hand, several rotational bands with large conversion coefficients can be strongly excited.

Numerical calculations for inner-shell ionization via internal conversion have been performed for the systems ${ }^{136} \mathrm{Xe}_{-}{ }^{238} \mathrm{U}$ and ${ }^{238} \mathrm{U}_{-}{ }^{238} \mathrm{U} .{ }^{42}$ The impact-parameter dependence obtained for the number of created vacancies per collision $P(b)$ differs 
considerably from that of direct ionization in the superheavy quasimolecule. Internal conversion leads to typically $0.2-0.3 \mathrm{~K}$ holes per central collision. Furthermore, we found for $P(b)$ a strong exponential decrease for larger impact parameters with falloff constants of $a \simeq 4 \mathrm{fm}$. The total cross sections of the nuclear process and direct ionization are comparable. Fortunately, by the Doppler shift of the characteristic $x$-ray lines, direct ionization $\left(\tau_{\mathrm{dir}}^{e^{-}} \sim 10^{-19} \mathrm{sec}\right)$ can be distinguished experimentally from internal conversion ionization $\left(\tau_{\text {conv }}^{e^{-}} \sim 10^{-10} \mathrm{sec}\right)$, which takes place when the ions are stopped in the target. ${ }^{79}$

On the other hand, the possibility of performing $\mathrm{x}$-ray spectroscopy ${ }^{29-31}$ of inner-shell electronic states in superheavy quasimolecules is strongly restricted by nuclear Coulomb excitation. For ${ }^{238} \mathrm{U}-{ }^{238} \mathrm{U}$ the nuclear photon spectrum exceeds the expected quasimolecular photon spectrum by at least two orders of magnitude. ${ }^{42}$ Only in the sys tem ${ }^{208} \mathrm{~Pb}+{ }^{208} \mathrm{~Pb}$ radiative transitions to the quasi molecular $K$ shell can be investigated experimentally. However, we have to stress that this conclusion does not concern the other molecular orbital transitions with only several keV energy. Here it has been already successfully demonstrated ${ }^{30}$ by the determination of peaks in the anisotropy of the quasimolecular radiation that electronic transition energies can be measured experimentally up to systems with $Z=Z_{1}+Z_{2} \sim 184$.

\section{TRANSITIONS BETWEEN BOUND STATES VIA RADIAL COUPLING}

In this section we consider the vacancy production probability of inner-shell electrons mediated by the radial coupling $\dot{R}\left\langle\phi_{f}|\partial / \partial R| \phi_{i}\right\rangle$ to higher bound states, which are supposed to be vacant ini tially $(t \rightarrow-\infty)$. In contrast to the direct excitation to the continuum, the following calculations include the assumption of an initial vacancy as parameter. Since it is not possible to solve the infinite set of coupled differential equations (9), we restrict our basis to the $1 s \sigma, 2 s \sigma, 3 s \sigma, 4 s \sigma$, and $5 s \sigma$ states. In order to save computer time it is also useful to transform Eq. (9) from $t$ to the parameter $\xi$ of the Rutherford trajectory:

$\frac{\partial a_{f}}{\partial \xi}=-\frac{\partial t}{\partial \xi} \sum_{i \dot{f}_{f}} a_{i}\left\langle\phi_{f}\left|\frac{\partial}{\partial t}\right| \phi_{i}\right\rangle \exp \left(i \int_{-\infty}^{t} d t^{\prime}\left(E_{f}-E_{i}\right)\right)$,

where from Eq. (4):

$$
\frac{\partial t}{\partial \xi}=\left(\frac{\mu a^{3}}{Z_{1} Z_{2} e^{2}}\right)^{1 / 2}(1+\epsilon \cosh \xi) .
$$

The diagonal matrix elements $\left\langle\phi_{f}|\partial / \partial t| \phi_{f}\right\rangle$ are purely imaginary and therefore vanish in the real representation of radial wave functions we have chosen.

The complex system of equations (51) has been solved $^{40}$ numerically by Hamming's modified predictor-corrector method. ${ }^{57}$ The number of channels was doubled by splitting them into real and imaginary parts. The sum of the amplitudes squared was normalized to unity:

$$
\sum_{n}\left|a_{n}\right|^{2}=1
$$

The most important modification compared with direct ionization to the continuum is the influence of the Pauli principle. In principle one has now to evaluate amplitudes for configurations described by Slater-determinants, instead of one-electron states. The number of coupled channels $N_{c}$ is

$$
N_{c}=N_{s} ! / N_{e} !\left(N_{s}-N_{e}\right) !,
$$

where $N_{s}$ denotes the number of one-electron basis states and $N_{e}$ denotes the number of electrons present. If there is only one electron or one hole, then the configuration equations are identical with the corresponding equations of the one-particle basis. Also the solution of complicator configurations can be performed by the reduction to the oneparticle basis. ${ }^{81}$

In general the results of coupled channel calculations for one electron show similar behavior as those for the direct excitation to the continuum. Let us first consider the ionization probability as a function of time during a collision of very heavy ions. On the incoming branch of the trajectory first contributions to $1 s \sigma$ ionization are given around $R \sim 100 \mathrm{fm}$, followed by a slight increase of $|a(t)|^{2}$. Just before the distance of closest approach is reached, a relative maximum in the vacancy-production rate appears and at $R=R_{\min }$ we find a sharp minimum. In the outgoing channel a steep increase occurs within a few $\mathrm{fm}$ change of distance. The maximum of $\left|a_{1 s \sigma}(t)\right|^{2}$ lies at $R=80$ $\mathrm{fm}$ in the U-U system and at $R=100 \mathrm{fm}$ in the $\mathrm{Pb}$ $\mathrm{Pb}$ system. The probability amplitude further continues like a damped oscillation. After the molecular $K$ shell is broken up at about $R=2000 \mathrm{fm}$ and approaches the atomic levels, the oscillations very quickly reach their constant asymptotic value. ${ }^{25,50}$

If we consider the probabilities $\left|a_{f}(t)\right|^{2}$ of dif ferent complicator configurations and not only of a single $1 s \sigma$ electron, this situation may change drastically. This typical feature of the coupled channel equations has its origin in the possibility of stepwise excitation and interference between different intermediate excitation paths. In Fig. 21 we show the probability for $1 s \sigma, 2 s \sigma$, and $3 s \sigma$ ionization as a function of the internuclear separa- 


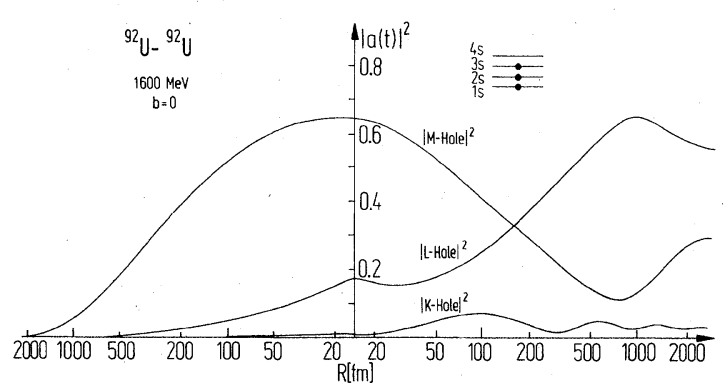

FIG. 21. Coupled channel analysis of vacancy formation during a head-on U-U collision with $E_{1 \mathrm{ab}}=1600 \mathrm{MeV}$. The number of created vacancies in plotted vs the internuclear separation $R$ during the collision. As initial configuration $(t \rightarrow-\infty)$ we assumed a vacant $4 s \sigma$ state and occupied $1 s \sigma, 2 s \sigma$, and $3 s \sigma$ states.

tion $R$, where initially $(t \rightarrow-\infty)$ we start from a vacant $4 s \sigma$ level. ${ }^{40}$ The calculations have been performed for a U-U head-on collision with $E_{\text {lab }}$ $=1600 \mathrm{MeV}$. We here employed true two-center energies and $\partial / \partial R$ matrix elements obtained under the assumption of pointlike nuclei. The asymptotic $K$-vacancy production probability amounts to several percent and is comparable with the direct ionization to the continuum. It is a remarkable. incident that the $3 s \sigma$ vacancy probability after the collision is smaller than that of the $2 s \sigma$ level.

In Fig. 22 we show for a few initial configura tions the number of $1 s \sigma$ vacancies created in the

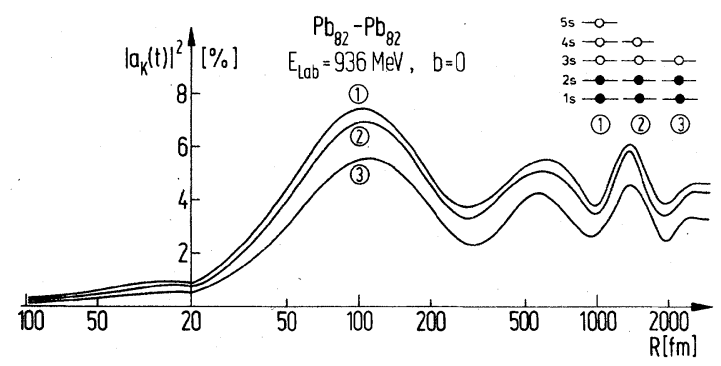

(a)

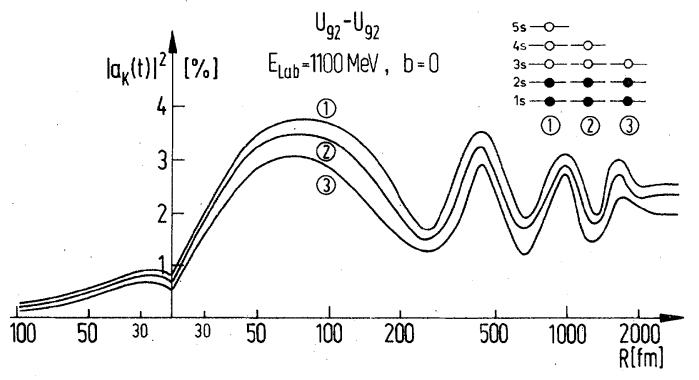

(b)

FIG. 22. Number of created $1 s \sigma$ vacancies during $\mathrm{Pb}-\mathrm{Pb}$ and $\mathrm{U}-\mathrm{U}$ head-on collisions. The different curves belong to different initial configurations.

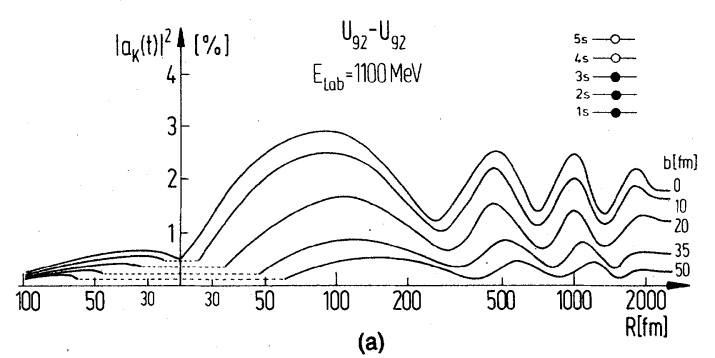

(a)

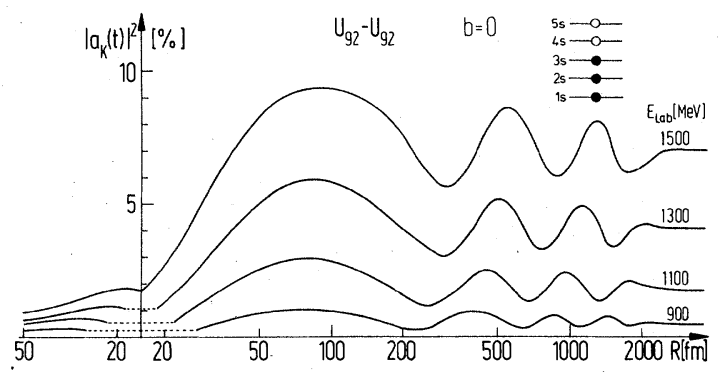

(b)

FIG. 23. Impact-parameter and bombarding energy dependence of $1 s \sigma$ vacancy formation during U-U collisions. In the coupled channel calculations the $4 s \sigma$ and $5 s \sigma$ states have been assumed to be vacant initially $(t \rightarrow-\infty)$.

$\mathrm{Pb}-\mathrm{Pb}$ and $\mathrm{U}-\mathrm{U}$ systems during the collision. Again we find as a main result several percent vacancy probability. The $1 s \sigma$ ionization is slightly larger when a larger number of empty final states are available. We have to stress that these results are based on the model assumption of vacant $3 s \sigma, 4 s \sigma$, and $5 s \sigma$ states. In Fig. 23 the timedependent $K$-vacancy probability is displayed as function of $R$ for various impact parameters $b$ and ion energies $E_{\text {lab. }}$. The $4 s \sigma$ and $5 s \sigma$ states have been assumed initially vacant. We obtain a strong falloff with increasing $b$, and decreasing $E_{\text {lab }}$ for the U-U collisions. To obtain $1 s \sigma$ ionization rates in the percent region, one has to choose $E_{\text {lab }}$ $>1000 \mathrm{MeV}$ and $b<20 \mathrm{fm}$.

Up to now we have treated only one-collision processes. This seems to be justified, because a $K$ vacancy in an $U$ atom lives only $\tau \sim 10^{-17} \mathrm{sec}$ and a second central collision within this time has negligible probability. Therefore, in the calculations described before only vacant high-lying states were assumed, whose ionization may occur in a preceding collision or at the beginning of the collision itself. Nevertheless, if we assume a double collision and choose as initial configuration the distribution of vacancies in that way as they originate from the first collision, the $K$-vacancy probability in a U-U head-on collision can be enhanced by more than $20 \%$, and strong oscillations already appear in the incoming channel. 
In a schematic model also the excitation to the positive continuum could be investigated in the framework of coupled channels with conservation of unitarity. ${ }^{40}$ Again we consider a U-U collision with $E_{\text {lab }}=1600 \mathrm{MeV}$. The emission of bound electrons takes place into an energy band centered around $E=1.4 \mathrm{~m}$, with a width of $\Gamma=1 \mathrm{MeV}$. Under this assumption the results of $1 s \sigma$ ionization obtained within time-dependent perturbation theory can be reproduced very well. On the first half of the trajectory we have a relatively strong increase of $\left|a_{1 s \sigma}(t)\right|^{2}$ reaching a maximum of $1.3 \%$ at $R=30$ $\mathrm{fm}$. At the distance of closest approach at $R_{\min }$ $=15 \mathrm{fm}$ we find $0.8 \%$ for the $1 s \sigma$ vacancy probability. Then again the rebound occurs, leading to an increase to $5.4 \%$ at $R=40 \mathrm{fm}$. The ionization probability reaches a constant final value of $2 \%$ at $R \sim 1000 \mathrm{fm}$.

The ion energy dependence of the $K$-vacancy production calculated in the framework of coupled channels can be taken from Fig. 24 for a special configuration. ${ }^{40}$ For the investigated U-U head-on collision, initially vacant $4 s \sigma$ and $5 s \sigma$ states have been assumed. Between $E_{\mathrm{lab}}=500$ and $1500 \mathrm{MeV}$ a risé by almost four orders of magnitude is observed.

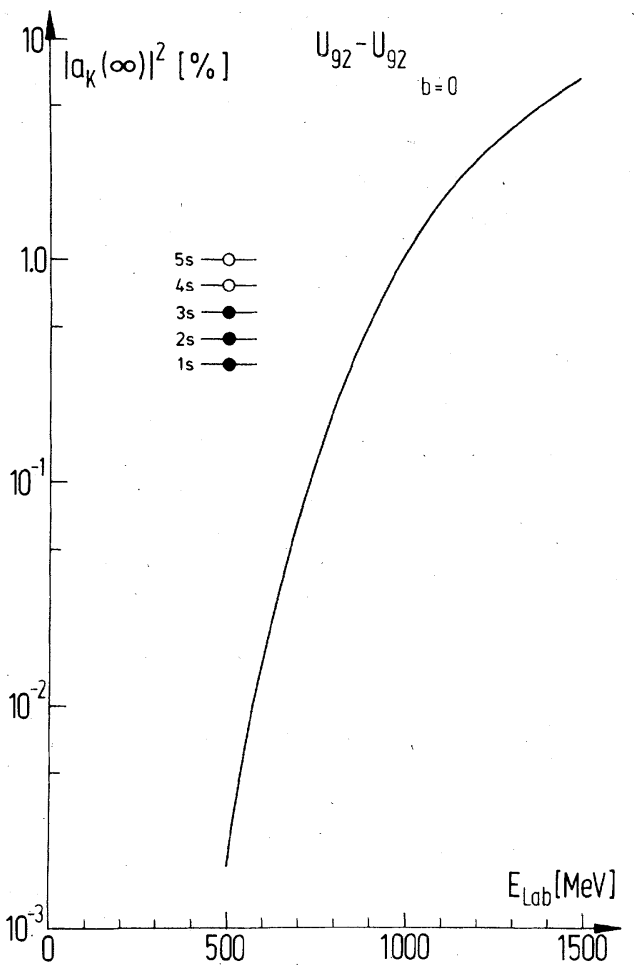

FIG. 24. Bombarding energy dependence of the number of created $1 s \sigma$ vacancies in U-U head-on collisions. In the coupled channel calculations the $4 s \sigma$ and $5 s \sigma$ states have been assumed to be vacant initially $(t \rightarrow-\infty)$.

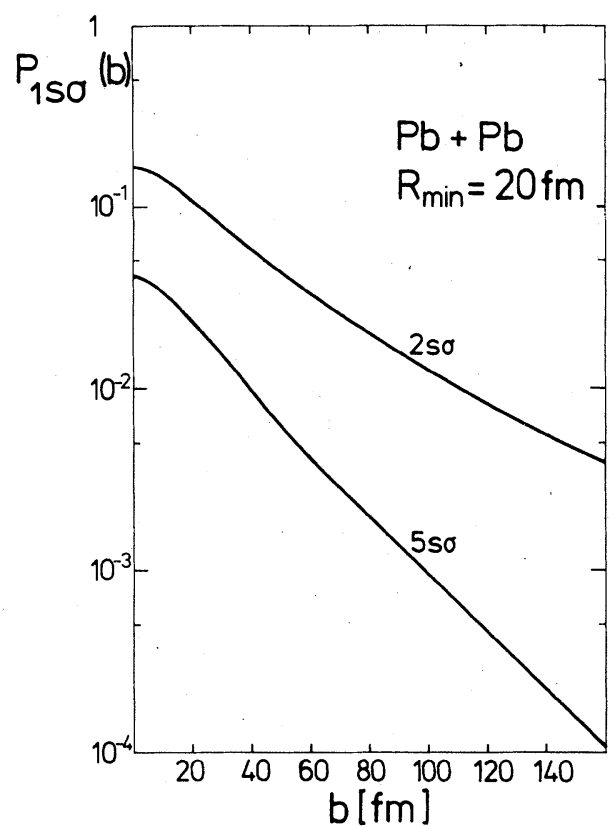

FIG. 25. Impact-parameter dependence of the number of created $1 s \sigma$ vacancies in a $\mathrm{Pb}-\mathrm{Pb}$ collision with $R_{\min }$ $=20 \mathrm{fm}$. In the coupled channel calculations denoted by $5 s \sigma$ the $1 s \sigma$ state up to the $5 s \sigma$ state have been included. The different curves belong to different initial configurations assuming one vacant state at $t \rightarrow-\infty$. The curve denoted by $2 s \sigma$ results from a two-state calculation. Here only transitions between the $1 s \sigma$ and $2 s \sigma$ state are considered.

Finally we show the impact-parameter dependence $P_{1 s \sigma}(b)$ for different initial configurations in Fig. 25. In this calculation energies and matrix elements of the monopole approximation were employed. The influence of finite nuclear size was taken into account. The ionization probability shows the familiar behavior. Altogether, it should be emphasized that slight modifications of matrix elements and energies may lead to large changes in $P(b)$.

\section{X. $\delta$ ELECTRONS}

For heavy-ion collisions with $Z_{1} \ll Z_{2}$ the highenergy tail of the continuum electron spectrum is dominated by ionization of the $K$ shell of the heavier atom. ${ }^{68-70,76}$ Compared with electronic states in the higher shells, usually the wave function of the $1 s$ state shows a sharp localization which corresponds to higher Fourier frequencies in the form factor. In this chapter we investigate the question of what information on inner-shell electronic states in superheavy quasimolecules can be obtained by measuring the $\delta$-electron spectrum.

In order to gain a first insight we plot in Fig. 26 the radial density distribution $|\psi r|^{2}=F^{2}+G^{2}$ for 


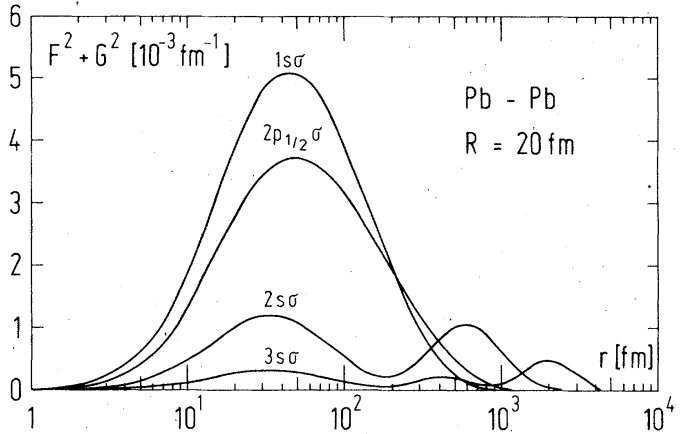

FIG. 26. Radial density distribution $|\psi r|^{2}=F^{2}+G^{2}$ for the $1 s \sigma, 2 p_{1 / 2} \sigma, 2 s \sigma$, and $3 s \sigma$ states in the $\mathrm{Pb}-\mathrm{Pb}$ quasimolecule at a separation $R=20 \mathrm{fm}$.

the $1 s \sigma, 2 p_{1 / 2} \sigma, 2 s \sigma$, and $3 s \sigma$ states in the $\mathrm{Pb}-\mathrm{Pb}$ quasimolecule at a separation $R=20 \mathrm{fm} .|\psi r|^{2}$ is very similar for the $1 s \sigma$ and $2 p_{1 / 2} \sigma$ states. This finding explains the equal size of the transitionmatrix elements, as discussed in Sec. VII. On the other hand, the Fourier frequencies necessary for ionization are much lower for the $2 p_{1 / 2} \sigma$ state than for the $1 s \sigma$ state. Therefore, without actually computing the $\delta$-electron spectrum a dominance of $2 p_{1 / 2} \sigma$ excitation can be expected even at large continuum energies $E$.

Numerical calculations have been carried out along the line of Eq. (11) in the monopole approxi mation. ${ }^{27,88}$ The continuum electron distributions $d \sigma / d E$ in the superheavy systems $Z=140,170$, and 190 resulting from $1 s \sigma$ and $2 p_{1 / 2} \sigma$ ionization are presented in Fig. 27. The distance of closest approach in head-on collisions $R_{\min }$, which determines the bombarding energy, was kept constant at $R_{\text {min }}=20 \mathrm{fm}$. $d \sigma / d E$ at $E \sim 1000 \mathrm{keV}$ increases as a function of total change by almost one order of magnitude from $Z=140$ to $Z=170$ and decreases again by a factor of 2 until $Z=190$. The electron spectrum is almost an exponential function falling typically by one decade over $\Delta E=400 \mathrm{keV}$. As already suspected, it results mainly from $2 p_{1 / 2} \sigma$ ionization for systems with $Z>140$, whereas for $Z=140$ the $1 s \sigma$ state generates an equally large contribution. For lower bombarding energies this qualitative behavior remains the same, aside from a slightly steeper slope of the electron distribution. Also an artificial shift of binding energies in a numerical simulation does not drastically change the relative size of the $p(\kappa=+1)$ and $s(\kappa=-1)$ wave contributions.

In Fig. 28 we present the $\delta$-electron spectrum observable in a $\mathrm{Pb}-\mathrm{Pb}$ collision with $R_{\min }=19.3$ fm. As initial bound states the $1 s \sigma, 2 s \sigma, 3 s \sigma$, and $2 p_{1 / 2} \sigma$ states are taken into account. The quasimolecular $K$ shell by far gives the smallest contribution. Experimentally we even expect

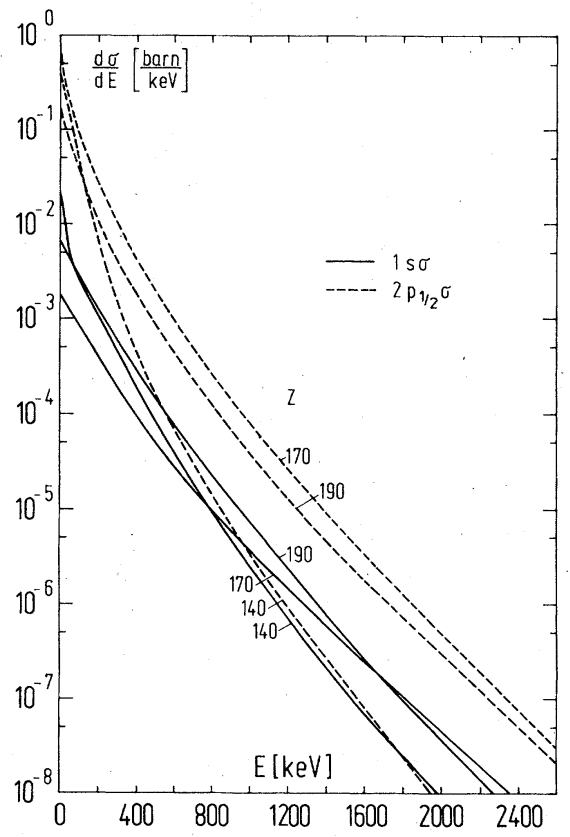

FIG. 27. $\delta$-electron spectrum resulting from $1 s \sigma$ and $2 p_{1 / 2} \sigma$ ionization in the superheavy systems $Z$ $=140,170$, and 190. The differential cross section $d \sigma /$ $d E$ with respect to final-state continuum energy is presented for fixed distance of closest approach in head-on collisions $R_{\min }=20 \mathrm{fm}$.

larger values for $d \sigma / d E$ than shown in Fig. 28, since we have neglected all the higher bound states. For instance, $3 p_{1 / 2} \sigma$ ionization leads to nearly the same spectrum as that of the $3 s \sigma$ state. Furthermore, corrections like electron screening lower the binding energies and therefore may increase the $\delta$-electron distribution by about $20 \%-$ $30 \%$.

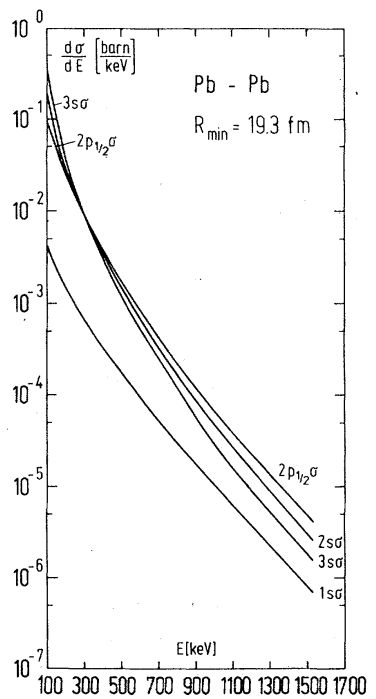

FIG. 28. $\delta$-electron spectrum in $\mathrm{Pb}-\mathrm{Pb}$ collision with $R_{\min }=19.3 \mathrm{fm}$. As initial bound states the $1 s \sigma$, $2 s \sigma, 3 s \sigma$, and $2 p_{1 / 2} \sigma$ states are considered. 
We conclude that a measurement of the electron spectrum may allow for a spectroscopy of the $L$ shell in superheavy quasimolecules. In order to deduce informations on the quasimolecular $K$ shell one has to perform a coincidence measurement of $\delta$ electrons with $K$ x rays of the heavier collision partner in asymmetric systems like $\mathrm{Xe}-\mathrm{Pb}$, where one can evade the $2 p \sigma-1 s \sigma$ vacancy sharing process. In the framework of the monopole approximation the angular distribution of the finalstate electrons is completely isotropic. The excitation process itself can be viewed as a breathing mode of the electronic cloud on the incoming and outgoing half of the Coulomb trajectory, which favors no special direction. We believe that this is a consequence of the approximation made, and that angular distributions would require a more sophisticated calculation.

How a spectroscopy with $\delta$ electrons could be performed can be exemplified in an analytical model for $1 s \sigma$ ionization in superheavy quasimolecules. ${ }^{62}$ According to this model the differential probability for $1 s \sigma$ excitation into a continuum state $E$ is given by

$$
\begin{aligned}
\frac{d P(b, E)}{d E}= & 4 \pi d_{0}^{2}(Z)\left(\frac{m_{e} c^{2}}{E}\right)^{\gamma(z)} \\
& \times \exp \left\{\frac{-4 \alpha(b) R_{\mathrm{min}}\left[E-E_{1 s \sigma}\left(R_{\mathrm{min}}\right)\right]}{\hbar v_{\infty}}\right\},
\end{aligned}
$$

with

$$
\alpha(b)=\left[2-2 a / R_{\min }\right]^{-1 / 2} .
$$

$R_{\min }$ and $a$ are defined in Eqs. (7) and (5), respectively, and $v_{\infty}$ is the ion velocity at infinity. Overall parametrizations of $d_{0}(z), \gamma(Z)$, and the energy eigenvalue $E_{1 s \sigma}(Z, R)$ have been published else where. ${ }^{60,62}$ For the $\mathrm{Xe}-\mathrm{Pb}(Z=136)$ system the values for pointlike nuclei are

$$
\begin{aligned}
& \gamma=2.18, d_{0}=0.003 \mathrm{keV}^{-1 / 2} \\
& E_{15 \mathrm{\sigma}}=-381 \mathrm{keV}\left(\frac{15 \mathrm{fm}}{R^{\prime}}\right)^{0.1162}+511 \mathrm{keV} .
\end{aligned}
$$

In Fig. 29 we have plotted the impact-parameter dependence (53) for $1 s \sigma$ ionization in a $\mathrm{Xe}-\mathrm{Pb}$ collision with $R_{\min }=16 \mathrm{fm}$. Several final-state energies $E$ are shown. Obviously, the high-energy electrons $(E \gtrsim 2 \mathrm{MeV})$ result from very close collisions $(b \leq 10 \mathrm{fm})$ only. However, the main feature of formula (53) is the one-to-one correspondence between the energy eigenvalue $E_{1_{s \sigma}}$ at $R_{\min }$ and the measurable probability for excitation to a fixed continuum state. Therefore, experimental data on the $\delta$-electron spectrum directly yield the $1 s \sigma$ binding energy at small separations.

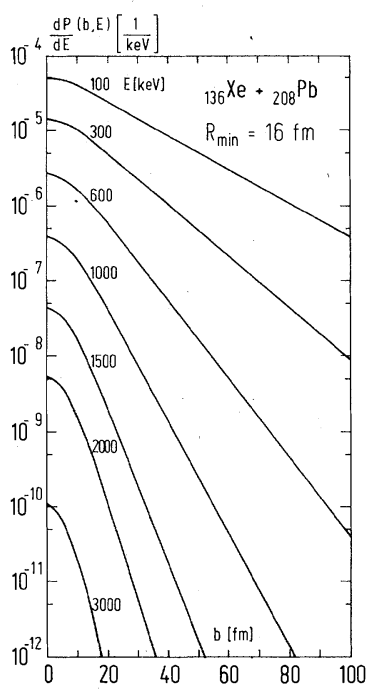

FIG. 29. Impact-parameter dependence of the differential probability of is $\sigma$ ionization with respect to final-state continuum energy. A Xe- $\mathrm{Pb}$ collision with $R_{\min }=16 \mathrm{fm}$ is considered. The calculations have been performed within an analytical model.

\section{INFLUENCE OF ROTATIONAL COUPLING ON $2 p_{1 / 2} \sigma$ VACANCY FORMATION}

As an example for ionization due to rotational coupling we consider vacancy creation in the quasimolecular $L$ shell of the $\mathrm{Pb}-\mathrm{Pb}$ system. For $1 s \sigma$ ionization rotational coupling effects are only of minor importance since the matrix elements $\left\langle 1 s \sigma\left|J_{x}\right| 2 s-\sigma\right\rangle,\left\langle 1 s \sigma\left|J_{x}\right| 3 s-\sigma\right\rangle,\left\langle 1 s \sigma\left|J_{x}\right| 3 d \pi\right\rangle$, etc., are negligible (see Figs. 6 and 8 ) for small separations $(R<500 \mathrm{fm})$ where most of the ioniza tion takes place. On the other hand, radial matrix elements exhibit a strong peak at small distances between both ions. However, the strong rotational coupling in coherence with the radial coupling between the substates of the quasimolecular $L$ shell leads to considerable vacancy production rates.

Greenberg et al.$^{36}$ have observed $\mathrm{Pb} K_{\alpha}$ radiation induced by collisions of $\mathrm{U}$ on $\mathrm{Pb}$, which is a clear indication of $2 p_{1 / 2} \sigma$ ionization in the superheavy quasimolecule $Z=174$. The impact-parameter dependence $P(b)$ of vacancy formation has been deduced by a kinematic analysis of Doppler broadened x-ray lines. $P_{\text {exp }}(b)$ shows a pronounced peak at $b=20 \mathrm{fm}$, which cannot be explained by ionization via radial coupling alone.

For the $\mathrm{Pb}-\mathrm{Pb}$ system we performed a coupled channel calculation, where it was assumed that the $2 p_{3 / 2} \pi$ state initially $(t \rightarrow-\infty)$ is vacant. The system of first-order differential equations (9) and (10) was solved numerically. In order to account for the Pauli principle one has to deal with configurations instead of single-electron states. Figure 30 shows the number of created vacancies $P_{f}=2\left|a_{f}(t)\right|^{2}$ as a function of the two-center distance $R$ for the assumed initial configuration in a $\mathrm{Pb}-\mathrm{Pb}$ collision with $4.7 \mathrm{MeV} / \mathrm{u}$. Just behind the 


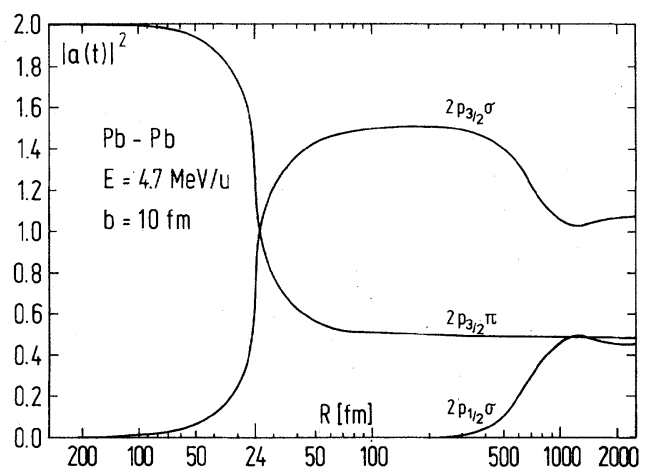

FIG. 30. Coupled channel analysis of vacancy formation in the quasimolecular $L$ shell during a $\mathrm{Pb}-\mathrm{Pb}$ collision with $R_{\min }=19.8 \mathrm{fm}$. The number of created vacancies are plotted vs two-center distance $R$. The $2 p_{3 / 2} \pi, 2 p_{3 / 2} \sigma$, and $2 p_{1 / 2} \sigma$ states are taken into account. As initial configuration $(t \rightarrow-\infty)$ we assumed a vacant $2 p_{3 / 2} \pi$ state. All rotational as well as radial couplings are included in the calculations.

distance of closest approach at $R_{\min }=19.8 \mathrm{fm}$, the $2 p_{3 / 2} \pi$ vacancies are transferred to the $2 p_{3 / 2} \sigma$ state via rotational coupling. Both levels are degenerate in the united atom limit. In the outgoing channel there occurs a strong radial coupling to the $2 p_{1 / 2} \sigma$ state at $R \sim 500 \mathrm{fm}$, so that the vacancy finally $(t \rightarrow \infty)$ may end up in the $K$ shell of one $\mathrm{Pb}$ nucleus. The impact-parameter dependence of this process is displayed in Fig. 31, where we added to these results the direct excitation to the continuum. ${ }^{25,41}$ The main features of the experimental data are reproduced very well. However, a dif ference lies in the location of the maximum, which

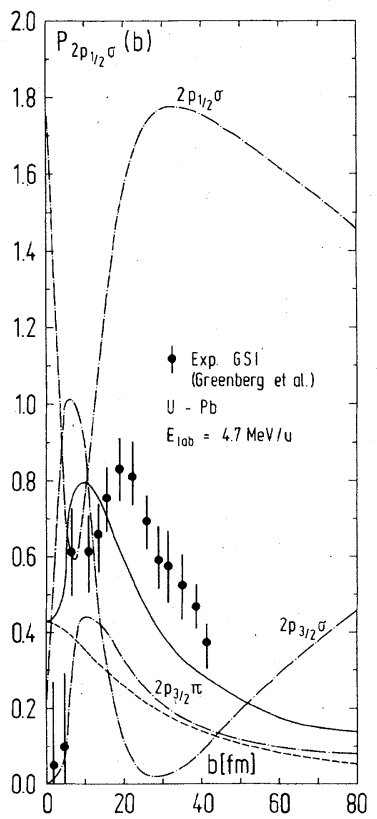

FIG. 31. Impact-parameter dependence of the number of created $2 p_{1 / 2} \sigma$ vacancies in a $\mathrm{Pb}-\mathrm{Pb}$ collision with $E_{1 \mathrm{ab}}=4.7 \mathrm{MeV} / \mathrm{u}$. The experimental data for the $\mathrm{U}-\mathrm{Pb}$ collision are taken from Ref. 36. Direct excitation to the continuum (dashed line) is compared with vacancy formation caused by rotational and radial couplings between the substates of the quasimolecular $L$ shell (dashed-dotted lines). Different initial configurations are considered in the coupled channel calculations characterized by the assumed vacant state at $t \rightarrow-\infty$. The solid line represents the sum of direct excitation to the continuum and of the curve where a vacant $2 p_{3 / 2} \pi$ state was assumed initially. is found to be at $b=10 \mathrm{fm}\left(90^{\circ}\right.$ scattering angle) in our theoretical calculation, whereas the experiment yields $b=20 \mathrm{fm}$. According to our computations a broad second maximum appears at $b$ $=200 \mathrm{fm}$, which gives a large contribution to the total ionization cross section. The other dashed curves in Fig. 31 are obtained by assuming dif ferent initial vacancy configurations. Only the coupling between bound states was considered.

\section{CONCLUSIONS}

For colliding systems with $Z_{1}+Z_{2} \varsigma 137$ a relativistic treatment of electronic states in quasimolecules is necessary. The relativistic effects manifest themselves in a considerable increase of the electronic density distribution near the charge centers (which is equivalent to high -momentum components of the wave functions), as well as in the increase of binding energies and finestructure splittings. These are first indications for the diving behavior into the negative energy continuum of inner-shell electrons. We found that ionization probabilities are enhanced by about four orders of magnitude due to relativistic effects ${ }^{58}$ compared with nonrelativistic estimates. The number of created $1 s \sigma$ vacancies per collision $P(b)$ amounts typically to several percent for central collisions and falls off exponentially with increasing impact parameter. For $K$-shell ionization in heavy ion collisions with $Z_{1}+Z_{2}<137$ we refer to the review article of Meyerhof and Taulbjerg. ${ }^{72}$

Our starting point for the theoretical description of the colliding system was the solution of the stationary two-center Dirac equation ${ }^{43,44}$ which provides binding energies of and coupling strengths between electronic states. An astonishing degree of simplification could be obtained by the application of the monopole approximation.

As a most important result we derived a way to experimentally determine electron binding energies in superheavy systems up to $Z=Z_{1}+Z_{2} \sim 190$. The method primarily employs the one-to-one correspondence between the impact-parameter dependence of ionization and the binding energy at the distance of closest approach on a given Coulomb trajectory. In particular, we found that the $Z$ dependence of ionization is a clear indication of strongly increasing binding energies for $Z_{1}+Z_{2}$ $z 164$. Meanwhile, $1 s \sigma$ binding energies up to $E_{1 s \sigma}=-600 \mathrm{keV}$ have been deduced from experimental data. ${ }^{74,86}$

Within model assumptions also vacancy creation via radial coupling between bound states has been investigated. Besides the magnitude of the transition probability, no principal difference to direct 
excitation to the continuum was found neither in bombarding energy nor in impact-parameter dependence. The influence of the strong rotational couplings between the substates of the quasimolecular $L$ shell has been examplified for the $\mathrm{Pb}-\mathrm{Pb}$ system. $P_{2 p 1 / 2 \sigma}(b)$ was modified drastically compared to results where only direct ionization to the continuum is considered.

Recent experimental data on ionization probabilities, ${ }^{36-39,75,82,84-85} \delta$-electron distributions, ${ }^{76}$ and quasimolecular $\mathrm{x}$ rays $^{31}$ indicate that the theoretical calculations correctly predicted the dependences on energy, impact parameter, and total nuclear charge. The experimental data are, however, typically a factor of 2-3 larger in magnitude than our theoretical results. This remaining discrepancy must be clarified in future calculations, possibly by improvements beyond the monopole approximation.

Note added in proof. Meanwhile we found that multiple excitations of inner-shell electrons in- crease the ionization probabilities by typically a factor of 2-3 essentially without changing the dependence on impact parameter, etc. Fair agreement between theoretical and experimental results is achieved by the inclusion of these multiple excitations between bound states and continuum states.

\section{ACKNOWLEDGMENTS}

We acknowledge fruitful discussions with J. Hamilton (Nashville), J. McGrory (Oak Ridge), J. Rafelski (CERN), J. Reinhardt (Frankfurt), R. Anholt (GSI), and P. Armbruster (GSI). We are grateful to G. Heiligenthal (Frankfurt) for making his coupled channel calculations available to us. This work has been supported by the Bundes ministerium für Forschung and Technologie (BMFT) and by the Gesellschaft für Schwerionenforschung (GST).
${ }^{1} \mathrm{G}$. Herrmann, Proceedings of the International Symposium on Superheavy Elements, Lubbock, Texas, 1978 (Pergamon, New York, 1978), p. 24.

${ }^{2}$ M. S. Freedman, F. T. Porter, and J. B. Mann, Phys. Rev. Lett. 28, 711 (1972).

${ }^{3}$ B. Fricke, J. B. Desclaux, and J. T. Waber, Phys. Rev. Lett. 28, 714 (1972).

${ }^{4}$ W. Pieper and W. Greiner, Z. Phys. 218, 327 (1969).

${ }^{5}$ I. Pomeranchuk and J. Smorodinsky, J. Phys. USSR $\underline{9}$, 97 (1945).

${ }^{6} \mathrm{~F}$. W. Werner and J. A. Wheeler, Phys. Rev. 109, 126 (1958).

${ }^{7}$ B. Fricke, W. Greiner, and J. T. Waber, Theor. Chim. Acta 21, 235 (1971).

${ }^{8}$ B. Fricke and G. Soff, At. Data Nucl. Data Tables 19 , 83 (1977).

${ }^{9}$ D. Rein, Z. Phys. 221, 423 (1969).

${ }^{10}$ B. Müller, J. Rafelski and W. Greiner, Nuovo Cimento A 18, 551 (1973).

${ }^{11}$ B. Mü̈ller, J. Rafelski, and W. Greiner, Z. Phys. 257, 62 (1972); 257, 183 (1972).

${ }^{12}$ Y. B. Zeldovich and V. S. Popov, Sov. Phys. Usp. 14, 673 (1972).

${ }^{13}$ M. Gyulassy, Phys. Rev. Lett. 33, 921 (1974).

${ }^{14}$ G. A. Rinker and L. Wilets, Phys. Rev. A 12, 748 (1975).

${ }^{15}$ K. T. Cheng and W. R. Johnson, Phys. Rev. A 14, 1943 (1976).

${ }^{16}$ J. P. Desclaux, At. Data Nucl. Data Tables 12, 311 (1973).

${ }^{17}$ B. Fricke and W. Greiner, Phys. Lett. B $\underline{30}, 317$ (1969).

${ }^{18}$ G. Soff, H.-J. Lustig, and B. Müller, J. Phys. G $\underline{3}$, 583 (1977).

${ }^{19}$ T. A. Carlson, C. W. Nestor, F. B. Malik, and R. C. Tucker, Nucl. Phys. A 135, 57 (1969).

${ }^{20}$ C. C. Lu, F. B. Malik, and T. A. Carlson, Nuc1. Phys. A 175,289 (1971).

${ }^{21}$ T. C. Tucker, L. D. Roberts, C. W. Nestor, T. A. Carlson, and F. B. Malik, Phys. Rev. 174, 118 (1968).
${ }^{22}$ C. C. Lu, T. A. Carlson, F. B. Malik, T. C. Tucker, and C. W. Nestor, At. Data 3, 1 (1971).

${ }^{23}$ T. A. Carlson and C. W. Nestor, At. Data Nucl. Data Tables 19, 153 (1977).

${ }^{24}$ T. H. Rihan, N. S. Aly, E. Merzbacher, B. Müller, and W. Greiner, Z. Phys. A 285, 397 (1978).

${ }^{25}$ G. Soff, W. Betz, G. Heiligenthal, J. Kirsch, B. Müller, J. Reinhardt, and W. Greiner, Fizika $\underline{\mathbf{9}}$, 721 (1977), Suppl. 4.

${ }^{26} \mathrm{G}$. Soff, B. Müller, and W. Greiner, Phys. Rev. Lett. 40,540 (1978).

${ }^{27}$ G. Soff, W. Betz, B. Müller, W. Greiner, and E. Merzbacher, Phys. Lett. A 65, 19 (1978).

${ }^{28}$ D. H. Jakubassa, Phys. Lett. A 58,163 (1976).

${ }^{29}$ J. Kirsch, W. Betz, J. Reinhardt, G. Soff, B. Müller, and W. Greiner, Phys. Lett. B 72, 298 (1978).

${ }^{30} \mathrm{~W}$. Wölfli, E. Morenzoni, Ch. Stoller, G. Bonani, and M. Stöckli, Phys. Lett. A 68, 217 (1978).

${ }^{31}$ W. E. Meyerhof, in Ref. 1, p. 415.

${ }^{32}$ R. Anholt, Z. Phys. A 288, 257 (1978).

${ }^{33} \mathrm{R}$. Anholt (unpublished).

${ }^{34} \mathrm{H}$. Hartung 'and B. Fricke, Z. Phys. A 288, 345 (1978).

${ }^{35}$ W. E. Meyerhof, Science 193, 375 (1976).

${ }^{36}$ J. S. Greenberg, H. Bokemeyer, H. Emling, E. Grosse, D. Schwalm, and F. Bosch, Phys. Rev. Lett. 39, 1404 (1977).

${ }^{37}$ J. R. McDonald, P. Armbruster, H. H. Behneke, F. Folkmann, S. Hagmann, D. Liesen, P. H. Mokler, and A. Warczak, Z. Phys. A 284, 57 (1978).

${ }^{38} \mathrm{R}$. Anholt, Phys. Rev. A 17, 834 (1978).

${ }^{39}$ R. Anholt and W. E. Meyerhof, Phys. Lett. A 64,381 (1978).

${ }^{40} \mathrm{G}$. Heiligenthal, thesis (Institut für Theoretische Physik, Universität Frankfurt, 1978) (unpublished).

${ }^{41} \mathrm{G}$. Heiligenthal, W. Betz, G. Soff, B. Müller, and W. Greiner, Z. Phys. A 285, 105 (1978).

${ }^{42} \mathrm{G}$. Soff, V. Oberacker, and W. Greiner, Phys. Rev. Lett. 41, 1167 (1978).

${ }^{43}$ B. Mülller, J. Rafelski, and W. Greiner, Phys. Lett. B 47, 5 (1973). 
${ }^{44} \mathrm{~B}$. Müller and W. Greiner, Z. Naturforsch. A $\underline{31}, 1$ (1976). ${ }^{45} \mathrm{~V}$. Oberacker and G. Soff, Z. Naturforsch. A 32,1465 (1977).

${ }^{46}$ W. Schäfer and G. Soff, Nuovo Cimento B 31, 250 (1976).

${ }^{47} \mathrm{~J}$. Rafelski, B. Müller, and W. Greiner, Lett. Nuovo Cimento 4 , 469 (1972).

${ }^{48}$ W. Schäfer, V. Oberacker, and G. Soff, Nucl. Phys. A 272,493 (1976).

${ }^{49} \mathrm{~J}$. Rafelski, Phys. Rev. C 13, 2086 (1976).

${ }^{50} \mathrm{~W}$. Betz, G. Heiligenthal, B. Müller, V. Oberacker, J. Reinhardt, W. Schäfer, G. Soff, and W. Greiner, in Predeal International Summer school, 1977, edited by V. Ceausescu and I. A. Dorobantu, Bucharest, Romania, p. 1.

${ }^{51} \mathrm{~J}$. Reinhardt and W. Greiner, Rep. Prog. Phys. $\underline{40}$, 219 (1977).

${ }^{52}$ B. Müller and W. Greiner, Acta Phys. Austriaca, Suppl. 18,153 (1977).

${ }^{53}$ C. Stoller, W. Wölfli, G. Bonani, M. Stöckli, and M. Suter, J. Phys. B 10, L347 (1977).

${ }^{54}$ K. H. Heinig, H. U. Jäger, H. Richter, H. Woitteneck, W. Frank, P. Gippner, K. H. Kaun, and P. Manfrass, J. Phys. B 10, 1321 (1977).

${ }^{55} \mathrm{~J}$. Rafelski and B. Müller, Phys. Lett. B 65, 205 (1976).

${ }^{56}$ J. Rafelski and B. Müller, Phys. Rev. Lett. 36,517 (1976).

${ }^{57} \mathrm{~A}$. Ralston and H. S. Wilf, Mathematical Methods for Digital Computers (Wiley, New York, 1960).

${ }^{58}$ W. Betz, B. Müller, G. Soff, and W. Greiner, Phys . Rev. Lett. 37, 1046 (1976).

${ }^{59}$ S. B. Schneiderman and A. Russek, Phys. Rev. 181, 311 (1969); V. SethuRaman, W. R. Thorson, and C. F. Lebeda, Phys. Rev. A 8, 1316 (1973); G. B. Schmid, Phys. Rev. A 15, 1459 (1977).

${ }^{60}$ G. Soff, J. Reinhardt, W. Betz, and J. Rafelski, Phys. Scr. 17, 417 (1978).

${ }^{61} \mathrm{E}$. M. Rose, Relativistic Electron Theory (Wiley, New York, 1961)

${ }^{62}$ B. Müller, G. Soff, W. Greiner, and V. Ceausescu, Z. Phys. A 285, 27 (1978).

${ }^{63}$ C. Foster, T. P. Hoogkamer, P. Woerlee, and F. W. Saris, J. Phys. B 9, 1943 (1976); W. E. Meyerhof, Phys. Rev. A 10, 1005 (1974).

${ }^{64} \mathrm{D}$. Burch, W. P. Ingalls, H. Wiemann, and R. Vandenbosch, Phys. Rev. A 10, 1245 (1974).

${ }^{65} \mathrm{~J}$. Reinhardt, V. Oberacker, G. Soff, B. Müller, and W. Greiner, Quantum electrodynamical effects in heavy ion collisions, International meeting on reactions of heavy ions with nuclei and synthesis of new elements, Dubna, 1977.

${ }^{66}$ R. Anholt, Phys. Rev. A 17, 976 (1978).
${ }^{67}$ G. Soff, B. Müller, and J. Rafelski, Z. Naturforsch. A 29, 1267 (1974).

${ }^{68}$ B.-H. Choi and E. Merzbacher, Phys. Rev. 177, 233 (1969).

${ }^{69}$ B.-H. Choi and E. Merzbacher, Phys. Rev. A 1 , 299 (1970).

${ }^{70} \mathrm{C}$. Kozhuharov, P. Kienle, D. H. Jakubassa and M. Kleber, Phys. Rev. Lett. 39, 540 (1977).

${ }^{71}$ D. R. Bates and R. McCaroll, Proc. R. Soc. A 245, 175 (1958).

${ }^{72}$ W. E. Meyerhof and K. Taulbjerg, Ann. Rev. Nucl. Sci. 27, 279 (1977).

${ }^{73}$ R. K. Smith, B. Müller, and W. Greiner, J. Phys. B $\underline{8}$, 75 (1975).

${ }^{74}$ H. H. Behncke, D.Liesen, S. Hagmann, P. H. Mokler, and P. Armbruster, Z. Phys. A 288, 35 (1978).

${ }^{75}$ R. Anholt, H.-H. Behncke, S. Hagmann, P. Armbruster, F. Folkmann, and P. Mokler, Z. Phys. A 289, 349 (1979).

${ }^{76}$ F. Bosch, H. Krimm, B. Martin, B. Povh, Th. Walcher and K. Traxel, Phys. Lett. B 78, 568 (1978).

${ }^{77}$ B. Müller, R. K. Smith, and W. Greiner, Phys. Lett. B 53, 401 (1975).

${ }^{78}$ G. Kraft, P. H. Mokler, and H. J. Stein, Phys. Rev. Lett. 33, 476 (1974).

${ }^{79} \mathrm{~J}$. S. Greenberg (private communication).

${ }^{80}$ D. R. Bates and D. A. Williams, Proc. Phys. Soc. Lond. 83,425 (1964).

${ }^{81} \mathrm{~J}$. Kirsch, thesis (Institut für Theoretische Physik, Universität Frankfurt, 1979) (unpublished).

${ }^{82}$ H.-H. Behncke, P. Armbruster, F. Folkmann, S. Hagmann, J. R. Macdonald, and P. H. Mokler, Z. Phys. A 289,333 (1979).

${ }^{83}$ P. A. Amundsen, J. Phys. B 11, L737 (1978).

${ }^{84}$ S. Hagmann, P. Armbruster, G. Kraft, P.-H. Mokler, and H.-J. Stein, Z. Phys. A 288, 353 (1978); A $\underline{290,} 25$ (1979).

${ }^{85}$ D. Liesen, P. Armbruster, H.-H. Behncke, and S. Hagmann, Z. Phys. A 288, 417 (1978).

${ }^{86}$ P. Armbruster, H. - H. Behncke, S. Hagmann, D. Liesen, F. Folkmann, and P. H. Mokler, Z. Phys. A 288, 277 (1978).

${ }^{87} \mathrm{~K} .-\mathrm{H}$. Wietschorke, B. Müller, W. Greiner, and G. Soff, J. Phys. B 12, L31 (1979).

${ }^{88}$ W. Greiner, B. Müller, and G. Soff, Phys。Lett. A $\underline{69}$, 27 (1978).

${ }^{89}$ P. Kaufmann and U. Wille, Z. Phys. A 279,259 (1976). 